\title{
A full Next to Leading Order study of direct photon pair production in hadronic collisions
}

\author{
T. Binoth, J.Ph. Guillet, E. Pilon, M. Werlen \\ Laboratoire d'Annecy-le-Vieux de Physique Théorique LAPTH * \\ B.P. 110, F-74941 Annecy-le-Vieux Cedex, France
}

\begin{abstract}
We discuss the production of photon pairs in hadronic collisions, from fixed target to LHC energies. The study which follows is based on a QCD calculation at full next-to-leading order accuracy, including single and double fragmentation contributions, and implemented in the form of a general purpose computer program of "partonic event generator" type. To illustrate the possibilities of this code, we present the comparison with observables measured by the WA70 and D0 collaborations, and some predictions for the irreducible background to the search of Higgs bosons at LHC in the channel $h \rightarrow \gamma \gamma$. We also discuss theoretical scale uncertainties for these predictions, and examine several infrared sensitive situations which deserve further study.
\end{abstract}

LAPTH-760/99

November 1999

*UMR 5108 du CNRS, associée à l'Université de Savoie. 


\section{Introduction}

The production of pairs of direct photons 1 with large invariant mass is the so called irreducible background for the search of the Higgs boson in the two photon decay channel in the intermediate mass range $80 \mathrm{GeV} \leq m_{h} \leq 140 \mathrm{GeV}$ at the forthcoming LHC. This background is huge and requires to be understood and quantitatively evaluated.

Beside this important motivation, this process deserves interest by its own. The production of such pairs of photons has been experimentally studied in a large domain of energies, from fixed targets [1, 2, 3] to colliders [田, 5, 6]. A wide variety of observables has been measured, such as distributions of invariant mass, azimuthal angle and transverse momentum of the pairs of photons, inclusive transverse momentum distributions of each photon, which offer the opportunity to test our understanding of this process.

The aim of this article is to present a study of di-photon hadroproduction based on a computer code of partonic event generator type, in which we account for all contributing processes consistently at next-to-leading order (NLO) accuracy. This article is organized according to the following outline. In section 2, we remind the basic theoretical ingredients, and present the method used to build the computer code developed for this study. Section 3 is dedicated to the phenomenology of photon pair production, with, a comparison with fixed target and collider experiments, then some predictions for LHC together with a discussion of theoretical scale uncertainties. The theoretical discussion about the present day limitations of our code is continued in section 4 . There we mention various infrared sensitive situations, which would deserve some more care, and for which the resummation of multiple soft gluon effects would be required, in order to improve the ability of our code to account for such observables. Section 5 gathers our conclusions and perspectives.

\section{Theoretical content and presentation of the method}

Let us first remind briefly the theoretical level of accuracy and limitations of works prior to the present one, in order to assess the improvements which we introduce. Then we present the method which we used to build our computer code DIPHOX.

\section{$2.1 \quad$ Theoretical content}

The theoretical understanding of this process relies on NLO calculations, initiated in [7].

The leading order contribution to di-photon reactions is given by the Born level process $q \bar{q} \rightarrow \gamma \gamma$ see for instance Diagram a. The computation of NLO contributions to it yields $\mathcal{O}\left(\alpha_{s}\right)$ corrections coming from the subprocesses $q \bar{q} \rightarrow \gamma \gamma g, g q$ (or $\bar{q}) \rightarrow \gamma \gamma q$ (or $\bar{q}$ ) and corresponding virtual corrections, see for example Diagrams b and c.

\footnotetext{
${ }^{1}$ The word "direct" means here that these photons do not result from the decay of $\pi^{0}, \eta$ at large transverse momentum. Direct photons may be produced according to two possible mechanisms: either they take part directly to the hard subprocess, or they result from the fragmentation of partons themselves produced at high transverse momentum in the subprocess; see sect. 2 .
} 


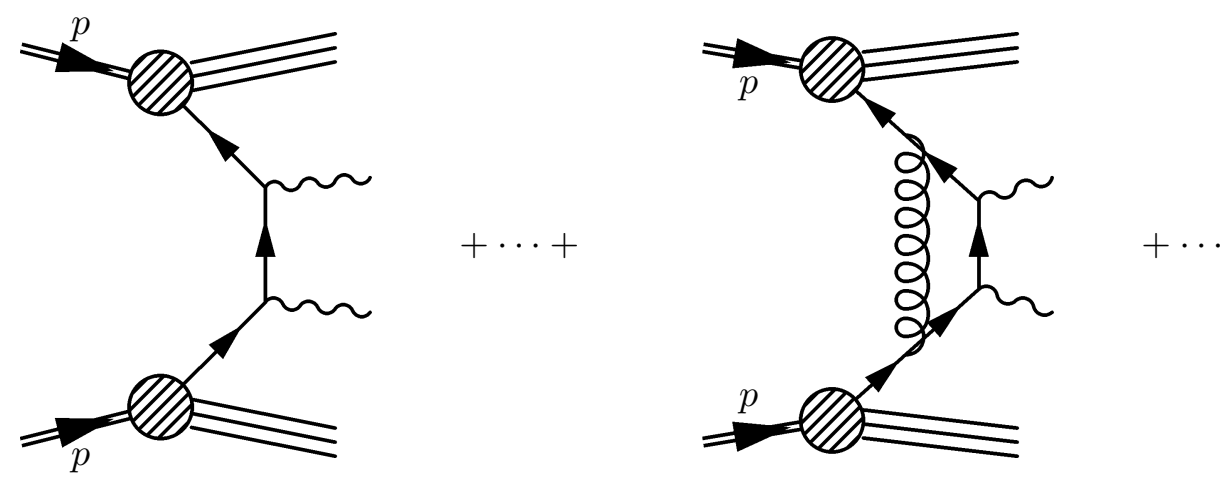

Diagram a

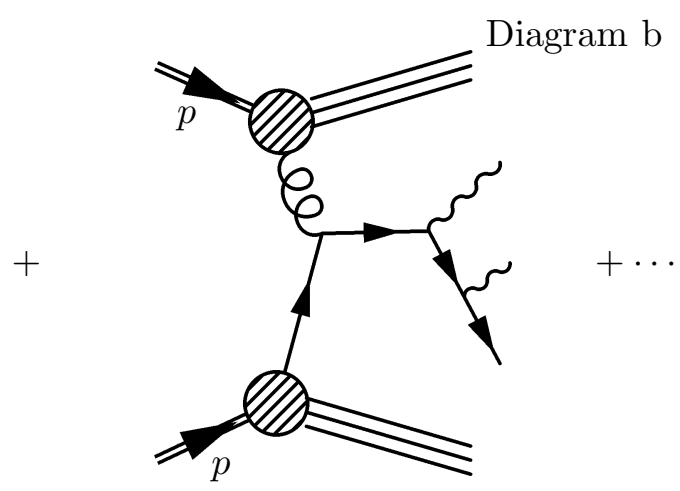

Diagram c

Yet it also yields the leading order contribution of single fragmentation type (sometimes called "Bremsstrahlung contribution"), in which one of the photons comes from the collinear fragmentation of a hard parton produced in the short distance subprocess, see for example Diagram d. From a physical point of view such a photon is most probably accompanied by hadrons. From a technical point of view this contribution comes from the appearance of final state collinear singularities in the higher order terms. These singularities are factorized and absorbed into quark and gluon fragmentation functions to a photon $D_{\gamma / q \text { or } g}\left(z, M_{f}^{2}\right)$ defined at some arbitrary fragmentation scale $M_{f}$. When the fragmentation scale $M_{f}$, chosen of the order of the hard scale of the subprocess, is large compared to any typical hadronic scale $\sim 1 \mathrm{GeV}$, these functions behave roughly as $\alpha / \alpha_{s}\left(M_{f}^{2}\right)$. Then a power counting argument tells that these contributions are asymptotically of the same order in $\alpha_{s}$ as the Born term $q \bar{q} \rightarrow \gamma \gamma$. What is more, given the high gluon luminosity at LHC, the $g q$ (or $\bar{q}$ ) initiated contribution involving one photon from fragmentation even dominates the inclusive production rate in the invariant mass range $80 \mathrm{GeV} \leq m_{\gamma \gamma} \leq 140 \mathrm{GeV}$. A consistent treatment of di-photon production at NLO thus requires that $\mathcal{O}\left(\alpha_{s}\right)$ corrections to these contributions be calculated also, see for example Diagrams e and f. They have not been incorporated in [7, 8, 9], and we compute them in the present work.
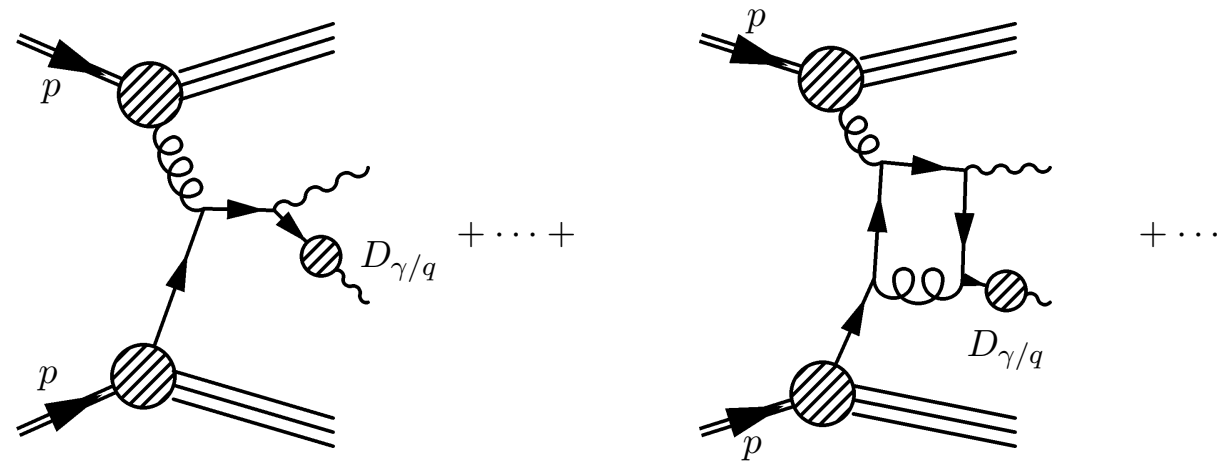

Diagram d

Diaoram e 


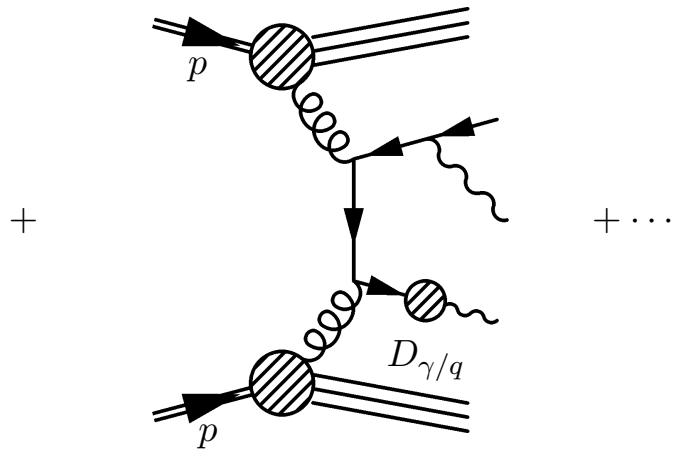

Diagram $\mathrm{f}$

The calculation of these corrections in their turn yields the leading order contribution of yet another mechanism, of double fragmentation type, see for example Diagram g. In the latter case, both photons result from the collinear fragmentation of a hard parton. In order to present a study of consistent NLO accuracy, NLO corrections to this double fragmentation contribution, see for example Diagrams $\mathrm{h}$ and $\mathrm{i}$, have to be calculated accordingly. This is also done in the present article.
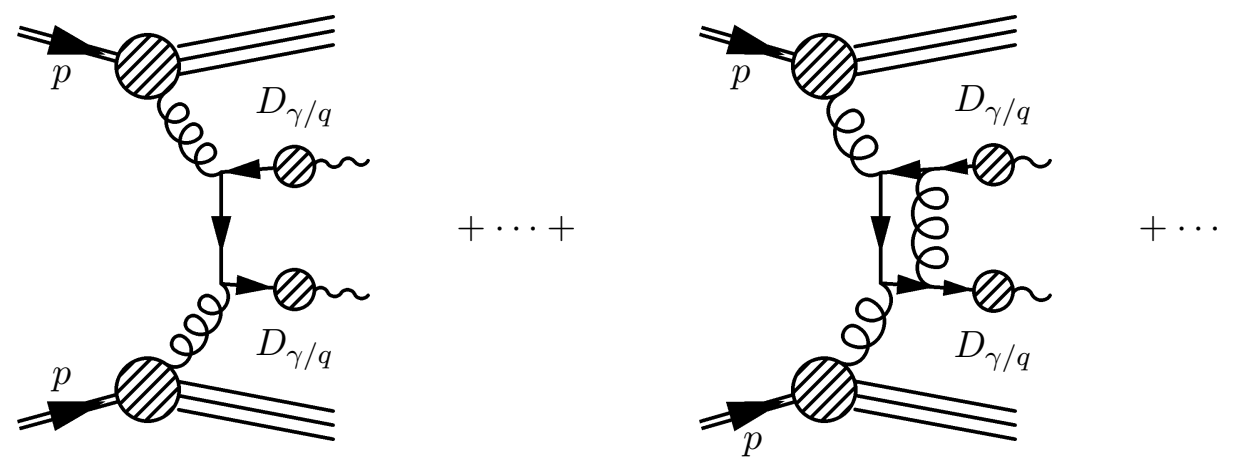

Diagram g

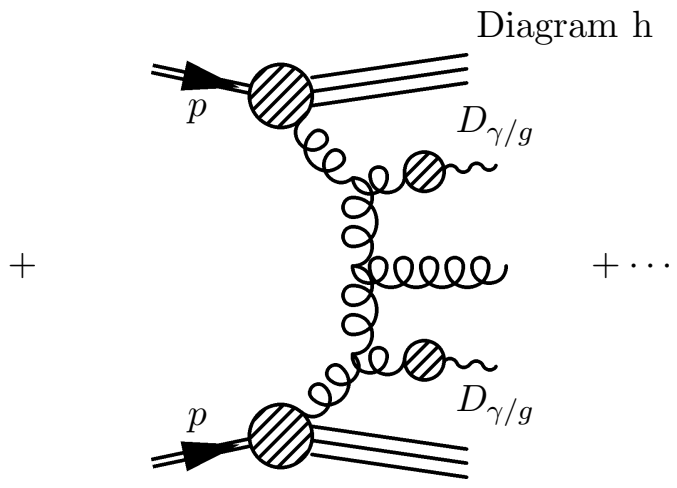

Diagram i

We call "two direct" the contribution given by the Born term plus the fraction of the NLO corrections which is free from final state collinear singularities. We call "one fragmentation" ("two fragmentation") the contribution involving one single fragmentation function (two fragmentation functions) of a parton into a photon. Let us add one more comment about the splitting into these three mechanisms. One must keep in mind that this distinction is schematic and ambiguous. We remind that it comes technically from the appearance of final state collinear singularities, which are factorized and absorbed into fragmentation functions at some arbitrary fragmentation scalef $M_{f}$. Each of the contributions associated with these three mechanisms thus depends strongly on this arbitrary scale. This dependence on $M_{f}$ cancels only in the sum of the three, so that this sum only is a physical observable. More precisely, a calculation of these contributions beyond leading order is required to obtain a (partial)

\footnotetext{
${ }^{2}$ More generally, the definition of the fragmentation functions rely on the choice of a given factorization scheme. In this work, we work in the $\overline{M S}$ factorization scheme. The fragmentation functions which we use are presented in 10 .
} 
cancelation of the dependence on $M_{f}$. Indeed this cancelation starts to occur between the higher order of the "two direct" contribution and the leading order of the "one fragmentation" term, and similarly between the "one-" and "two fragmentation" components respectively. This is actually one of the first motivations of the present work. Thus, even though it may be suggestive to compare the respective sizes and shapes of the separate contributions for a given choice of scale, as will be done in subsect. 3.2.1, we emphasize that only their sum is meaningful.

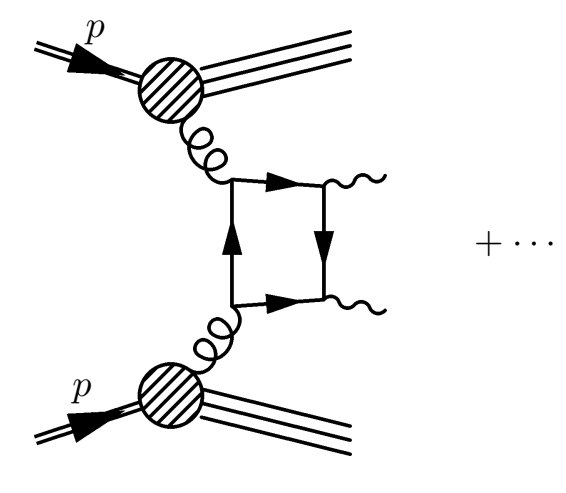

\section{Diagram j}

Beyond this, the $\mathcal{O}\left(\alpha_{s}^{2}\right)$ so-called box contribution $g g \rightarrow \gamma \gamma$ through a quark loop is also included, see for example Diagram j. Strictly speaking it is a NNLO contribution from the point of view of power counting. However in the range of interest at LHC for the search of the Higgs boson, the gluon luminosity is so large compared with the quark and antiquark one, that it nearly compensates the extra powers of $\alpha_{s}$, so as to yield a contribution comparable with the Born term. For this reason, it has been included in previous works, and will be in the present one as well. We define the "direct" contribution as the sum "two direct" + box.

Actually one should notice, firstly, that other NNLO gluon-gluon initiated processes, such as the collinear finite part of $g g \rightarrow \bar{q} q \gamma \gamma$ have been ignored ${ }^{\beta}$, although they could also be large. Secondly one should also even worry about the next correction to the box, because the latter may be quite sizeable. Such a possibility is suggested by the situation occuring to the first correction to the effective vertex $g g \rightarrow h$ computed in [11], who showed that it reaches generically about $50 \%$ of the one-loop result. Moreover, this box contribution is the leading order of a new mechanism, whose spurious (factorization and renormalization) scale dependences are monotonic, and only higher order corrections would partly cure this problem and provide a quantitative estimate. This tremendous effort has not been carried out yet, although progresses towards this goal have been achieved recently [12, 13, 14].

\subsection{Presentation of the method}

In [7], a dedicated calculation was required for each observable. Since then more versatile approaches have been developed, which combine analytical and Monte-Carlo integration techniques [8], [15]. They thus allow the computation of several observables within the same calculation, at NLO accuracy, together with the incorporation of selection/isolation cuts at the partonic level in order to match the various cuts used by the experimental collaborations as faithfully as possible. The studies of [8] and of [9] rely on such an approach. Let us briefly describe the one which we use here.

\subsubsection{Phase space slicing}

Within the combined analytical and Monte-Carlo approach, two generic well known methods can be used to deal with infrared and collinear singularities which are met in the calculation of inclusive cross

\footnotetext{
${ }^{3}$ The collinear divergent parts of these $2 \rightarrow 4$ processes have been already taken into account in the NLO corrections to the "one fragmentation" contribution and leading order "two fragmentation" components respectively.
} 
sections: the phase space slicing method [16] and the subtraction method [17]. In this work we use a version of the phase space slicing method 15. For a generic reaction $1+2 \rightarrow 3+4+5$ two particles of the final state, say 3 and 4 , have a high $p_{T}$ and are well separated in phase space, while the last one, say 5 , can be soft, or collinear to either of the four others. The phase space is sliced in the following way:

\section{- Part I}

The norm $p_{T} 5$ of transverse momentum of the particle 5 is required to be less than some arbitrary value $p_{T m}$ taken to be small compared to the other transverse momenta. This cylinder supplies the infrared, and initial state collinear singularities. It also yields a small fraction of the final state collinear singularities.

- Part II a

The transverse momentum vector of the particle 5 is required to have a norm larger than $p_{T m}$, and to belong to a cone $C_{3}$ of small radius $R_{t h}$ around the direction of particle 3 , defined by $\left(y_{5}-y_{3}\right)^{2}+\left(\phi_{5}-\phi_{3}\right)^{2} \leq R_{t h}^{2}$. This cone contains the remaining singularities appearing when 5 is collinear to 3 .

- Part II b

The transverse momentum vector of the particle 5 is required to have a norm larger than $p_{T m}$, and to belong to a cone $C_{4}$ of small radius $R_{t h}$ around the direction of particle 4 , defined by $\left(y_{5}-y_{4}\right)^{2}+\left(\phi_{5}-\phi_{4}\right)^{2} \leq R_{t h}^{2}$. This cone contains the remaining singularities appearing when 5 is collinear to 4 .

- Part III

The transverse momentum vector of the particle 5 is required to have a norm larger than $p_{T m}$, and to belong to neither of the two cones previously defined. This slice yields no divergence, and can thus be treated directly in 4 dimensions.

The collinear and soft singularities which appear on parts I, IIa and IIb are regularized by dimensional continuation from 4 to $d=4-2 \epsilon, \epsilon<0$. The $d$-dimensional integration over the kinematic variables (transverse momentum, rapidity and azimuthal angles) of the particle 5 is performed analytically over the parts I, IIa and IIb. For an extended presentation of the details and corresponding explicit formulas, we refer to 15 .

After combination with the corresponding virtual contributions, the infrared singularities cancel, and the remaining collinear singularities which do not cancel are factorized and absorbed in parton distribution or fragmentation functions. We call the resulting quantities pseudo cross sections for $2 \rightarrow 2$ and quasi $2 \rightarrow 2$ processes, whose detailed content is given in the Appendix. The word "pseudo" reminds that they are not genuine cross sections, as the $2 \rightarrow 2$ and quasi $2 \rightarrow 2$ contributions are not positive in general. These pseudo cross sections, as well as the transition matrix elements on part III, are further used to sample kinematic configurations, in the framework of a partonic event generator.

\subsubsection{Partonic event generator}

For practical purposes, a partonic event generator has been built for diphoton production including all the mechanisms: the "direct", "one-" and "two fragmentation". Each mechanism is treated separately. Firstly, the contribution of a given mechanism to the integrated cross section is calculated with the integration package BASES [18]. At this stage, some kinematic cuts (e.g. on the rapidity of the two photons, on their transverse momenta, etc.) may be already taken into account. Then, for the $2 \rightarrow 2$ contributions, the quasi $2 \rightarrow 2$ contributions, and the inelastic contributions on the part III of the phase space, partonic events are generated with SPRING [18] with a weight \pm 1 depending on the sign of the integrand at this point of the phase space 1 . All the events are subsequently stored

\footnotetext{
${ }^{4}$ This trick circumvents the fact that SPRING works only with positive integrands, while the pseudo cross sections are not positive.
} 
into a NTUPLE [19]. Finally these NTUPLES are histogramed at will.

During this projection some more refined cuts can be applied such as isolation criteria on the photons. Indeed, the collider experiments ( $S p \bar{p} S$, Tevatron, and the forthcoming LHC) do not measure inclusive photons. Instead, the experimental event selection of direct photons requires the use of an isolation criterion, in order to reject the huge background of secondary photons produced in the decays of mainly $\pi^{0}$ and $\eta$. Schematically, a photon is isolated if, inside a cone centered around the photon direction, defined in the rapidity and azimuthal angle plane by

$$
\left(y-y_{\gamma}\right)^{2}+\left(\phi-\phi_{\gamma}\right)^{2} \leq R^{2}
$$

the amount of hadronic transverse energy deposited is smaller than some value $E_{T \text { max }}$ fixed by the experiment. In our calculation, the transverse energy deposited in the cone may come from the residue of the fragmentation, from the parton 5 (which never fragments into photons) or from both. Any summation of potentially large logarithms which may come from this new constraint of the phase space is not performed. This issue will be discussed again in subsect. 4.2.

Let us state more clearly what we mean by partonic event generator. Since the events associated to the $2 \rightarrow 2$ and quasi $2 \rightarrow 2$ contributions have a negative weight, this code, properly speaking, is not a genuine event generator on an event-by-event basis. By events, we mean final state partonic configurations. For a given event, the informations stored into the NTUPLE are the 4-momenta of the outgoing particles; their flavours: parton (i.e. quark or gluon) or photon; in the fragmentation cases, the longitudinal fragmentation variable(s) associated with the photon(s) from fragmentation; and, for practical purpose, a label which identifies the type of pseudo cross section $(2 \rightarrow 2$, quasi $2 \rightarrow 2$, inelastic) which produced the event stored. Notice that in the fragmentation cases, all but the longitudinal information on the kinematics of the residue of the collinear fragmentation is lost. Hence this type of program does not provide a realistic, exclusive portrait of final states as given by genuine event generators like PYTHIA [20] or HERWIG [21]. On the other hand, the latter are only of some improved leading logarithmic accuracy. It is more precisely a general purpose computer program of Monte-Carlo type, whose virtue is the computation of various correlation observables within the same calculation, at NLO accuracy.

\section{Phenomenology}

In this section, we adopt a LHC oriented presentation. We start with a brief comparison of our NLO calculations with WA70 and D0 data for illustrative purpose. We then show some predictions for LHC in the invariant mass range $80 \mathrm{GeV} \leq m_{\gamma \gamma} \leq 140 \mathrm{GeV}$ corresponding to the Higgs boson search through $h \rightarrow \gamma \gamma$. We discuss the ambiguities plaguing these predictions due to the arbitrariness in the choices of the renormalization scale $\mu$, of the initial state factorization scale $M$ (which enters in the parton distribution functions), and of the fragmentation scale $M_{f}$.

\subsection{Comparison with experimental data}

\subsubsection{Fixed target data}

A comparison between the diphoton differential cross section versus each photon's transverse momentum measured by the WA70 collaboration [1] and our NLO postdiction is shown on Fig. [1. There, one can see the respective magnitude of the various contributions for the scale choice $M=M_{f}=\mu=$ $\lambda\left(p_{T}\left(\gamma_{1}\right)+p_{T}\left(\gamma_{2}\right)\right)$, with $\lambda=0.275$, evaluated with the ABFOW parton distribution functions [22].

\footnotetext{
${ }^{5}$ The WA70 collaboration requires the transverse momenta of the photons to be larger than $3 \mathrm{GeV}$ and $2.75 \mathrm{GeV}$ respectively. However for convenience we first implemented a symmetric cut on the $p_{T}$ of each photon: $p_{T} \geq 2.75 \mathrm{GeV}$ at the level of the Monte Carlo generation of photon pairs. On the other hand in the ABFOW parametrizations, the factorization scale $M^{2}$ has to be larger than $2 \mathrm{GeV}^{2}$. Taking $\lambda \geq .275$ ensures that $M^{2}$ is always above $2 \mathrm{GeV}^{2}$, unlike with $\lambda=1 / 4$.
} 
The "one fragmentation" contribution is one order of magnitude below the "two direct" contribution. The "two fragmentation" contribution is even smaller and negligible here. The smallness of these contributions is the reason why previous works [7, 8] described this observable reasonably well too, despite the absence of higher order corrections to the fragmentation contributions there.

Correlations between the two photons: the distribution of the variable $z=-\mathbf{p}_{T}\left(\gamma_{1}\right) \cdot \mathbf{p}_{T}\left(\gamma_{2}\right) / p_{T}^{2}\left(\gamma_{1}\right)$, the $p_{T}$ imbalance, the distribution of the azimuthal angle between the two photons, the $p_{\text {out }}$ distribution, and the transverse momentum distribution of diphotons, have been measured also by the WA70 collaboration [2]. These distributions, which are infrared sensitive near the elastic boundary of the spectrum (e.g. $q_{T} \rightarrow 0$ or $\phi_{\gamma \gamma} \rightarrow \pi$ ) or near a critical point (e.g. $z=1$ ) are moreover quite sensitive to non perturbative effects appearing in the resummed part, over a wide part of the spectrum covered by the measurements. Consequently we do not present any comparison of these data points with the approximation of fixed order accuracy of this work; nor will we discuss the scale ambiguities at fixed target energies.

\subsubsection{Tevatron collider data}

A preliminary study of diphotons events in the central region $\left(\left|y\left(\gamma_{1,2}\right)\right|<1.0\right)$ has been recently performed by the D0 collaboration [6]. The experimental cuts on the photons transverse energies, respectively $p_{T}\left(\gamma_{1}\right)>14 \mathrm{GeV}$ and $p_{T}\left(\gamma_{2}\right)>13 \mathrm{GeV}$, are raised in our parton level NLO theoretical calculations to $p_{T}\left(\gamma_{1}\right)>14.9 \mathrm{GeV}$ and $p_{T}\left(\gamma_{2}\right)>13.85 \mathrm{GeV}$ respectively, to take into account smearing from the electromagnetic calorimeter resolution. The actual isolation cuts used experimentally are quite more complicated than the schematic criterion (11), and cannot be faithfully implemented at the partonic level. We instead simulated them in our NLO calculation by requiring that the accompanying transverse partonic energy be less than $E_{T \max }=2 \mathrm{GeV}$ in a cone $R=0.4$ about each photon. We then studied the effect of varying $E_{T \text { max }}$ from 1 to $3 \mathrm{GeV}$ in the calculated cross-section, in order to roughly estimate both the stability of our modelization, and the effect of smearing due to hadronic calorimeter resolution. This turned out to have a less than $4 \%$ effect on our estimation.

The MRSG set of parton distributions functions [23] is used, with the scales $M=M_{f}=\mu=$ $\left(p_{T}\left(\gamma_{1}\right)+p_{T}\left(\gamma_{2}\right)\right) / 4$. The different contributions: "direct", "one-" and "two fragmentation" for the above scale choice, are shown for the diphoton differential cross sections vs. the transverse momentum of each photon (Fig. 2), the diphoton mass (Fig. 3), and for the transverse momentum of photon pairs (Fig. (4) and the azimuthal angle between the photons (Fig. 5). These last two distributions are well known to be controlled by multiple soft gluon emission near the elastic boundary of the spectrum, $q_{T} \rightarrow 0$ and $\phi_{\gamma \gamma} \rightarrow \pi$ respectively. Consequently, the accuracy of any fixed-order calculation, including the present one, is not suited to study such observables in these respective ranges. More on this issue will be commented in the next section. On the other hand a NLO calculation is expected to be predictive for the tails of these distributions away from the infrared sensitive region.

The data are reasonably described, taking into account a correlated systematic error for events in which the $p_{T}$ of both photons is above $20 \mathrm{GeV}$. This correlated systematic error due to the background evaluation affects obviously the three highest $p_{T}$ points of the transverse energy spectrum, as well as the three highest points of the diphoton mass spectrum. With the scale choice used, the double fragmentation yields a tiny contribution.

We do not present any analysis of the various scale dependences for Tevatron. Such a discussion is proposed for LHC in the next section. Yet let us mention that, at Tevatron, the energy scale is lower and the relevant values of $x$ are somewhat higher than at LHC. Consequently, the renormalization scale dependence is slightly sharper, on the other hand the factorization scale dependence is somewhat flatter than at LHC. Nevertheless the situation at Tevatron is expected to be qualitatively similar to the one at LHC. 


\subsection{Predictions for $\mathrm{LHC}$}

We now discuss some results computed with the kinematic cuts from the CMS and ATLAS proposals [24], namely $p_{T}\left(\gamma_{1}\right)>40 \mathrm{GeV}, p_{T}\left(\gamma_{2}\right)>25 \mathrm{GeV},\left|y\left(\gamma_{1,2}\right)\right|<2.5$, with $80 \mathrm{GeV} \leq m_{\gamma \gamma} \leq 140 \mathrm{GeV}$, and using the MRST2 set of parton distribution functions [25] and the fragmentation functions of [10].

\subsubsection{Scale ambiguities}

We first consider the invariant mass distribution of diphotons, in absence of isolation cuts, cf. Fig. 6] in order to illustrate the strong dependence of the splitting into the three contributions, "direct", "one-" and "two fragmentation", on the scale chosen, as we warned in subsect. 2.1. In both choices of scales displayed the "one fragmentation" contribution dominates, but the hierarchy between "direct" and "two fragmentation" contributions is reversed from one choice to the other. With the choice of scales $M=M_{f}=\mu=m_{\gamma \gamma} / 2$, the "one fragmentation" is more than twice larger than the "direct" one, and the "two fragmentation" is the smallest. On the other hand, with the other choice $M=M_{f}=\mu=2 m_{\gamma \gamma}$, the "one fragmentation" contribution is three to five times larger than the "two fragmentation" component, and more than one order of magnitude above the "direct" one" On the other hand the total contribution seems rather stable.

Yet the arbitrariness in the choices of the various scales still induces theoretical uncertainties in NLO calculations. In the following we actually do not perform a complete investigation of all three scale ambiguities independently with search for an optimal region of minimal sensitivity. At the present stage, we limit the study to an estimation of the pattern and magnitude of their effect on our results. We show how the scale ambiguities affect our prediction for the invariant mass distribution. We consider both the case without isolation (Fig. (7) and the isolated case with $E_{T \text { max }}=5 \mathrm{GeV}$ inside $R \leq 0.4$ (Fig. 8). For the present purpose, the virtue of the actual values the isolation parameters used here is to strongly suppress the fragmentation contributions hence the associated $M_{f}$ dependence. We compare four different choices of scales: two choices along the first diagonal $\mu=M=M_{f}=m_{\gamma \gamma} / 2$ and $\mu=M=M_{f}=2 m_{\gamma \gamma}$; and two anti diagonal choices, $\mu=m_{\gamma \gamma} / 2 ; M=M_{f}=2 m_{\gamma \gamma}$ and $\mu=2 m_{\gamma \gamma} ; M=M_{f}=m_{\gamma \gamma} / 2$. We do not a perform a separate study of fragmentation scale dependence. Yet the latter can be indirectly estimated by comparing the results of the isolated case, where the fragmentation components, thereby the corresponding fragmentation scale dependence, are strongly suppressed, with the situation in the non isolated case, where especially the "one fragmentation" contribution is quite large, and the "two fragmentation" not negligible, so that the issue of fragmentation scale dependence matters.

When scales are varied between $m_{\gamma \gamma} / 2$ and $2 m_{\gamma \gamma}$ along the first diagonal $\mu=M=M_{f}$, the NLO results for the invariant mass distribution appear surprisingly stable, since they change by about $5 \%$ only. Alternatively, anti-diagonal variations of $\mu$ and $M=M_{f}$ in the same interval about the central value $m_{\gamma \gamma}$ lead to a variation still rather large (up to $20 \%$ cf. Fig. 7 and Fig. 8). This is because variations with respect to $\mu$ and $M$ are separately monotonous but act in opposite ways. When $\mu$ is increased, $\alpha_{s}\left(\mu^{2}\right)$ hence the NLO corrections decrease?. On the other hand the relevant values of momentum fraction of incoming partons are small, $\sim \mathcal{O}\left(10^{-3}\right.$ to $\left.10^{-2}\right)$ so that the gluon and sea quark distribution functions increase when $M$ is increased. In the isolated case, this leads to a monotonous increase of the "direct" component, over a large band of the invariant mass range considered, as $M$ is

\footnotetext{
${ }^{6}$ The oscillations of the latter result from numerical cancelations between rather large contributions of opposite signs

${ }^{7}$ In processes for which the lowest order is proportional to some power $\alpha_{s}^{n}, n \geq 1$, an explicit $\mu$ dependence appears in the next-to-leading order coefficient function, which partially compensates the (large) $\mu$ dependence in $\alpha_{s}\left(\mu^{2}\right)$ weighting the lowest order. Unlike this, in the "two direct" component which dominates the cross section when a drastic isolation is required, the lowest order involves no $\alpha_{s}$. This leads to a rather small $\mu$ dependence, since the latter starts only at NLO. On the other hand, the $\mu$ dependence occurs only through the monotonous decrease of the $\alpha_{s}\left(\mu^{2}\right)$ weighting the first higher order correction: there is no partial cancelation of $\mu$ dependence. Such cancelation would start only at $\mathcal{O}\left(\alpha_{s}^{2}\right)$, i.e. at NNLO. The mechanism is more complicated in presence of fragmentation components, and the situation becomes mixed up between all components when the severity of isolation is reduced.
} 
increased, cf. Fig. 9, which is induced in particular by the monotonous increase of the box contribution. Scale changes with respect to $\mu$ and $M$ turn out to nearly cancel against each other along the first diagonal but add up in the other case. Actually, the stability along the first diagonal is accidental.

In conclusion, the $\mu, M$ dependences are thus not completely under control yet at NLO in the kinematic range considered. On the opposite, the account for the NLO corrections to the fragmentation components provides some stability with respect to $M_{f}$ variations about orthodox choices of the fragmentation scale.

The issue of $\mu$ dependence of less inclusive observables, such as the tails of the $q_{T}$ or $\phi_{\gamma \gamma}$ distributions are the same for the invariant mass distribution. This is because the tails of these distributions is purely given by the NLO corrections and dominated by the $\mathcal{O}\left(\alpha_{s}\right)$ corrections of the "two direct" component. On the other hand, the $M$ dependence is a bit larger, so is the combined uncertainty on the theoretical results for these distributions, cf. Fig. 10 and Fig. 11.

\subsubsection{Effect of isolation}

We now consider the effect of isolation on the various contributions. As expected, isolation reduces the diphoton production rate, with respect to the inclusive case, cf. Fig. 12. More precisely, severe isolation requirements like $E_{T \max }=5 \mathrm{GeV}$ inside a cone $R=0.4$ suppress the "one fragmentation" component, which dominated the inclusive rate, by a factor 20 to 50 , and kill the "two fragmentation" contribution completely.

However this net result hides a rather intricate mechanism, cf. Fig. 13 vs. Fig. 6, by which the "two direct" contribution turns out to be increased! Surprising as it may seem at first sight, this effect has the following origin. Higher order corrections to the "two direct" component involve in particular the two subprocesses $\bar{q} q \rightarrow \gamma \gamma g$ and $g q \rightarrow \gamma \gamma q$ (where $q$ is a quark or an antiquark). The first one yields a positive contribution. On the other hand, the collinear safe part of the second one yields a contribution which is negative, and larger in absolute value than the previous one in the inclusive case, as was already seen in [7]. Isolation turns out to suppress more the higher order corrections from the second mechanism than from the first one, so that the NLO isolated "two direct" contribution is larger than the inclusive one. Yet, the "fragmentation" contributions are suppressed more than the "two direct" one is increased, so that the sum of all contributions is indeed decreased, with respect to the inclusive case. Once again, one has to remember that the splitting into the three mechanisms depend, non only on the factorization scale, but more generally factorization scheme. This arbitrari-

ness generates such counterintuitive offsprings; in a final state factorization scheme different than the $\overline{M S}$ scheme, the various components, especially the "two direct" one, may be separately affected by isolation cuts in a different way. This once more illustrates the danger of playing with these unphysical quantities separately.

A more detailed analysis of the dependence of NLO estimations of various observables on the isolation cut parameters, especially on $E_{T \max }$ will be given in a forthcoming publication. We will also come back to this issue, regarding infrared sensitivity, in subsect. 4.3 .

\section{Infrared sensitive observables of photon pairs and soft gluon di- vergences.}

Being based on a fixed, finite order calculation, our computer code is not suited for the study of observables controlled by multiple soft gluon emission, and has to be improved in this direction. 
Among these infrared sensitive observables, one may distinguish the following examples, most of which would require an improved account of soft gluon effects.

\subsection{Infrared sensitivity near the elastic boundary}

\subsubsection{The transverse momentum distribution of photon pairs $d \sigma / d q_{T}$ near $q_{T}=0$}

Both in the inclusive and isolated cases, this distribution is an infrared sensitive observable, controlled by the multiple emission of soft and collinear gluons. This well known phenomenon has been extensively studied for the corresponding observable in the Drell-Yan process [26]. A loss of balance between the contribution of real emission, strongly suppressed near this exclusive phase space boundary, and the corresponding virtual contribution, results in large Sudakov-type logarithms of $m^{2} / q_{T}^{2}$ ( $m$ being the invariant mass and and $q_{T}$ the transverse momentum of the photon pair - the heavy vector boson in the Drell-Yan case) at every order in perturbation. In order to make sensible predictions in this regime, these Sudakov-type logarithms have to be resummed to all orders.

The treatment of the "two direct" and box contributions is similar to the well-known Drell-Yan process, and has been carried out recently by [27] at next-to-leading logarithmic accuracy in the framework tailored by Collins, Soper and Sterman [28]. On the other hand, the fragmentation contributions do not diverge order by order when $q_{T} \rightarrow 0$. Indeed, in the "one fragmentation" case,

$$
\begin{aligned}
\operatorname{parton}_{1}+\operatorname{parton}_{2} & \rightarrow \gamma_{1}+\text { parton }_{3} \\
\text { parton }_{3} & \rightarrow \gamma_{2}+X
\end{aligned}
$$

the NLO contribution to the hard subprocess (2) yields a double logarithm of the form

$$
\sim \alpha_{s} \ln ^{2}\left\|\mathbf{p}_{T}\left(\gamma_{1}\right)+\mathbf{p}_{T}\left(\operatorname{parton}_{3}\right)\right\|
$$

when $\| \mathbf{p}_{T}\left(\gamma_{1}\right)+\mathbf{p}_{T}\left(\right.$ parton $\left._{3}\right) \| \rightarrow 0$. However the extra convolution associated with the fragmentation (3) involves an integration over the fragmentation variable $p_{T}\left(\gamma_{2}\right) / p_{T}\left(\right.$ parton $\left._{3}\right)$ which smears out this integrable singularity. The "two fragmentation" contribution involves two such convolutions, hence one more smearing.

\subsubsection{The distribution of photon-photon azimuthal angle difference $d \sigma / d \phi_{\gamma \gamma}$ near $\phi_{\gamma \gamma}=\pi$}

This distribution is another interesting infrared sensitive observable, measured by several experiments both at fixed target and collider energies [2, 5, 6], though less discussed in the literature from the theoretical side. The regime $\phi_{\gamma \gamma} \rightarrow \pi$ includes back-to-back photons, a set of configurations which lie at the elastic boundary of the phase space. This case differs from the previous one for two reasons. Firstly, not only the "two direct" contribution diverges order by order when $\phi_{\gamma \gamma} \rightarrow \pi$, but also both "one-" and "two fragmentation" contributions diverge as well, as can be seen on Fig. 5. Indeed, consider the example of the "one fragmentation case", cf. equations 2 and 3. Selecting $\phi_{\gamma \gamma} \rightarrow \pi$ emphasizes the configurations with $\phi\left(\right.$ parton $\left._{3}\right)-\phi\left(\gamma_{1}\right) \rightarrow \pi$, so that all the emitted partons besides parton 3 have to be collinear to either of the incoming or outgoing particles, and/or soft, which yields double logarithms

$$
\sim \alpha_{s} \ln ^{2}\left[\pi-\left(\phi\left(\operatorname{parton}_{3}\right)-\phi\left(\gamma_{1}\right)\right)\right]
$$

associated with each of the hard partons 1,2,3 - plus single logarithms as well. For the observable $d \sigma / d \phi_{\gamma \gamma}$ near $\phi_{\gamma \gamma}=\pi$, the integral involved in the convolution of the hard subprocess with the fragmentation functions does not smear these logarithmic divergences, since the fragmentation variable $p_{T}\left(\gamma_{2}\right) / p_{T}\left(\right.$ parton $\left._{3}\right)$ is decoupled from the azimuthal variable $\phi\left(\right.$ parton $\left._{3}\right)$ which is equal to $\phi\left(\gamma_{2}\right), \gamma_{2}$ and parton 3 being collinear. A similar observation holds for the "two fragmentation" component. Moreover, in both fragmentation cases, soft gluons may couple to both initial and final state hard emitters. The resulting color structure of the emitters is more involved than in the "two direct" case, 
and especially more complicated in the "two fragmentation" case as shown in some recent works [29]. This would make any resummation quite intricate beyond leading logarithms.

Let us notice that both fragmentation components make $d \sigma / d \phi_{\gamma \gamma}$ diverge also when $\phi_{\gamma \gamma} \rightarrow 0$. The increase of the fragmentation contributions in the lower $\phi_{\gamma \gamma}$ range is the trace of this divergence, cf. Fig. 5.

\subsection{An infrared divergence inside the physical region.}

In the case of photons isolated with the standard fixed cone size criterion of eqn. (1), a new problem appears in the $q_{T}$ distribution. This problem does not concern the region $q_{T} \rightarrow 0$; still it has to do with infrared and collinear divergences. This can be seen on Fig. 14, which shows the observable $d \sigma / d q_{T}$ vs. $q_{T}$ for isolated photon pairs, computed at NLO accuracy. The computed $q_{T}$ distribution turns out to diverge when $q_{T} \rightarrow E_{T \max }$ from below. Notice that the critical point $E_{T \max }$ is located inside the physical region. The phenomenon is similar to the one discovered in [30] in the production of isolated photons in $e^{+} e^{-}$annihilation, and whose physical explanation has been given in 31 following the general framework of [32]. It is a straightforward exercise to see that the lowest order "one fragmentation" contribution has a stepwise behaviour, as noticed in [9]. Indeed, at this order, the two photons are back-to-back. $E_{T \text { had }}$ being the transverse hadronic energy deposited in the cone about the photon from fragmentation, the conservation of transverse momentum implies at this order that $E_{T h a d}=q_{T}$. The corresponding contribution to the differential cross section $d \sigma / d q_{T}$ thus takes the schematic form:

$$
\left(\frac{d \sigma}{d q_{T}}\right)^{(1 \text { fragm, } L O)}=f\left(q_{T}\right) \Theta\left(E_{T \max }-q_{T}\right)
$$

According to the general analysis of [32], the NLO correction to $d \sigma / d q_{T}$ has a double logarithmic divergence at the critical point $q_{T}=E_{T \max }$. The details of this infrared structure are very sensitive to the kinematic constraints and the observable considered. In the case at hand, at NLO, $d \sigma / d q_{T}$ gets a double logarithm below the critical point, which is produced by the convolution of the lowest order stepwise term above, with the probability distribution for emitting a soft and collinear gluon:

$$
\left(\frac{d \sigma}{d q_{T}}\right)^{(1 \mathrm{fragm}, N L O)} \simeq-f\left(q_{T}\right) \frac{\alpha_{s}}{2 \pi} C \ln ^{2}\left(1-\frac{q_{T}^{2}}{E_{T \max }^{2}}\right) \Theta\left(E_{T \max }-q_{T}\right)+\cdots
$$

where $C$ is a color factor, $C_{F}$ or $N_{c}$ according to whether the soft collinear gluon emitter is a quark (antiquark) or a gluon. More generally, at each order in $\alpha_{s}$, up to two powers of such logarithms will appear, making any fixed order calculation diverge at $q_{T}=E_{T \text { max }}$, so that the spectrum computed by any fixed order calculation is unreliable in the vicinity of this critical value. An all order resummation has to be carried out if possible in order to restore any predictability. A correlated step appears also in the "two direct" contribution at NLO, in the bin about $q_{T}=E_{T \max }$. A detailed study of these infrared divergences will be presented in a future article.

No such divergence appears in the $q_{T}$ distribution of photon pairs presented in [9]. The non appearance of the double logarithmic divergence there comes from the fact that the latter pops out only at NLO, while the authors of [9] compute the "one fragmentation" component at lowest order. Furthermore, the stepwise lowest order "one fragmentation" contribution to the $q_{T}$ distribution is replaced in [9] by the result of the Monte Carlo simulation of this component using PYTHIA [20]. A quantitative comparison is thus difficult to perform?.

\footnotetext{
${ }^{8}$ In practice, the $q_{T}$ spectrum is sampled into bins of finite size, and the distribution represented on Fig. 14 is averaged on each bin. Since the logarithmic singularity is integrable, no divergence is actually produced. However when the bin size is shrunk, the double logarithmic branch appears again.

${ }^{9}$ Such a comparison involves two issues.

The first aspect concerns the infrared sensitivity below the critical point. When the scale of $\alpha_{s}$ in the Sudakov factor of the fragmenting quark is chosen to be the transverse momentum of the emitted gluon with respect to the emitter,
} 
It can be noticed that the divergence at $q_{T}=E_{T \max }$ is not visible on Fig. 1 . This is because in this case, the critical point $E_{T \max }$ in the $q_{T}$ spectrum where the theoretical calculation diverges is too close to the other singular point $q_{T}=0$, given the binning used. The two singularities contribute with opposite signs in these bins and a numerical compensation occurs, resulting in no sizeable effect. Yet the problem is only camouflaged. A similar smearing appears also at LHC energies for a stringent isolation cut, cf. Fig. 10.

\subsection{Reliability of NLO calculations with stringent isolation cuts}

Let us add one more comment concerning NLO partonic predictions with very stringent isolation cuts. In such calculations, the isolation cuts act on the products of the hard subprocess only. On the other hand, in an actual LHC event, a cut as severe as $E_{T \max }=2.5 \mathrm{GeV}$ inside a cone $R=0.3$ or 0.4 will be nearly saturated by underlying events and pile up.

This means that such an isolation cut actually allows almost no transverse energy deposition from the actual hadronic products of the hard process itself. This may be most suitable experimentally, and one may think about simulating such an effect safely in an NLO partonic calculation by using an effective transverse energy cut much more severe than the one experimentally used. However, requiring that no transverse energy be deposited in a cone of fixed size about a photon is not infrared safe, i.e. it would yield a divergent result order by order in perturbation. This implies that NLO partonic calculations implemented with finite but very stringent isolation cuts in a cone of fixed finite size would lead to unreliable results, plagued by infrared instabilities involving large logarithms of $E_{T \max }$. What is more, these infrared nasties would not be located at some isolated point in the diphoton spectrum (like some elastic boundary or some critical point, as in the previous subsection), but instead they would extend over its totality, even for observables such as the invariant mass distribution. The issue of an all order summation of these logarithms of $E_{T \max }$ would have to be investigated in this case.

\section{Conclusions and perspectives}

We presented an analysis of photon pair production with high invariant mass in hadronic collision, based on a perturbative QCD calculation of full NLO accuracy. The latter is implemented in the form of a Monte Carlo computer programme of partonic event generator type, DIPHOX. The postdictions of this study are in reasonable agreement with both WA70 fixed target, and preliminary D0 collider data, in the kinematical range where the NLO approximation is safe, namely away from the elastic boundary of phase space. Yet more will be learnt from the final analysis of the Tevatron data, and even more so after the Tevatron run II in the perspective of the LHC. It will then be worthwhile to perform a more complete phenomenological study.

This notwithstanding, there remains room for improvements. A first improvement will be to take into account multiple soft gluon effects in order to calculate infrared sensitive observables correctly. Another improvement will concern a more accurate account of contributions beyond NLO, associated namely with the gluon-gluon initiated subprocess. Among those are the NNLO corrections, and even the two loop, so-called double box correction to $g g \rightarrow \gamma \gamma$, which may be quantitatively important at

the parton shower not only reproduces the fragmentation function of a parton into a photon to the collinear leading logarithmic approximation, but it also provides an effective resummation of soft gluons effects to infrared and collinear leading logarithmic accuracy. (This would not be true if, instead, the scale of $\alpha_{s}$ in the Sudakov factor were the virtuality of the emitter). This ensures that the distribution does not diverge from below at the critical point, but rather tends to a finite limit.

The second issue concerns the shape of the tail of this contribution above the critical point. Indeed, energy-momentum conservation at each branching makes the parton shower generate also contributions in the region $q_{T}>E_{T \text { max }}$, which is forbidden at lowest order. These contributions would be classified in a beyond leading order calculation as higher order corrections. Unlike in a fixed order calculation however, they provide only a partial account of such corrections, but to arbitrary high order. The accuracy of these terms is thus uneasy to characterize, and a quantitative comparison between PYTHIA and any fixed order calculation is difficult to perform. 
LHC for the background to Higgs search.

A better understanding of the effects of isolation, and their interplays with infrared problems is also required. This concerns the $q_{T}$ distribution near the critical point $q_{T}=E_{T \text { max }}$ induced by isolation even when $E_{T \max }$ is not small; this concerns also the status of partonic predictions when $E_{T \max }$ is chosen very small. Alternatively it would be interesting to explore the properties of different isolation criteria, such as, for example, the one invented recently by Frixione [33]. Concerning these last two items, approaches relying on beyond leading order partonic level calculations, and full event generators like PYTHIA or HERWIG will be complementary.

Acknowledgments We acknowledge discussions with J. Wormesley on the D0 data, J. Owens on theory vs. data comparisons, and C. Balazs about the theoretical ingredients inside the RESBOS code. We thank F. Gianiotti, P. Petroff, E. Richter-Was and V. Tisserand for discussions concerning the Atlas Proposal. This work was supported in part by the EU Fourth Training Programme "Training and Mobility of Researchers", Network "Quantum Chromodynamics and the Deep Structure of Elementary Particles", contract FMRX-CT98-0194 (DG 12 - MIHT).

\section{A Technical details on the two photon production}

In this appendix, we give some details on the method used to deal with infrared and soft divergences. For a complete presentation, we refer to [15]. The most complicated kinematics happens in the two fragmentation mechanism. Only the two fragmentation contribution will be treated in this appendix, the kinematics of the other cases can be simply deduced replacing the fragmentation function by a Dirac distribution:

$$
D_{\gamma / k}\left(x, M_{f}^{2}\right)=\delta(1-x)
$$

At the hadronic level, the reaction $H_{1}\left(K_{1}\right)+H_{2}\left(K_{2}\right) \rightarrow \gamma\left(K_{3}\right)+\gamma\left(K_{4}\right)+X$ is considered with:

$$
\begin{aligned}
K_{1} & =\frac{\sqrt{S}}{2}(1, \mathbf{0}, 1) \\
K_{2} & =\frac{\sqrt{S}}{2}(1, \mathbf{0},-1) \\
K_{3} & =K_{T 3}\left(\cosh y_{3}, \boldsymbol{n}_{3}, \sinh y_{3}\right) \\
K_{4} & =K_{T 4}\left(\cosh y_{4}, \boldsymbol{n}_{4}, \sinh y_{4}\right)
\end{aligned}
$$

where

$$
\boldsymbol{n}_{3}^{2}=\boldsymbol{n}_{4}^{2}=1
$$

The cross section of the preceeding reaction is the sum of the following parts.

- The part I (cf. section 2.2.1) contains infrared and initial state collinear singularities. Once these divergences have been removed (cancelled against virtual divergences or absorbed into the bare distribution functions), this part generates two types of finite terms.

- The first type, of infrared origin, has the same kinematics as the lowest order (LO) terms and is given in A.4 Pseudo cross section for the infrared and virtual parts .

- The second type, of collinear origin, has an extra integration over the center of mass energy of the hard scattering, as compared to LO kinematics. For this reason, it is called quasi $2 \rightarrow 2$. It is given in A.2 Pseudo cross sections for the initial state collinear parts.

- The parts IIa and IIb contain the final state collinear singularities. Once these divergences have been absorbed into the bare fragmentation functions, the remaining finite terms $\mathbb{T}$ involve

\footnotetext{
${ }^{10}$ More precisely, a part of the final state collinear divergences comes from the cylindrical region (part I). The associated finite terms are added to the ones coming from part IIa (resp. part IIb) cf. equations (A.10) and (A.11).
} 
an extra integration over the relative momentum of the collinear partons, as compared to LO kinematics. They are thus called quasi $2 \rightarrow 2$ as well. They are given in A.3 Pseudo cross sections for the final state collinear parts.

- The part III has no divergences. It is given in A.1 Cross section for real emission.

\section{A.1 Cross section for real emission}

The cross section is parametrized on the following form:

$$
\begin{aligned}
\sigma= & C_{i j} \int d y_{3} \int d y_{4} \int d K_{T 3} \int d K_{T 4} \\
& \times\left[\int_{x_{3 \min }}^{1} \frac{d x_{3}}{x_{3}} \int_{p_{T m}}^{p_{T 5 m a x}} d p_{T 5} p_{T 5} \iint_{\Omega_{35}-C_{3}} d \phi_{35} d y_{5}\right. \\
& \times p_{T 3} D_{\gamma / k}\left(x_{3}, M_{f}^{2}\right) \frac{D_{\gamma / l}\left(x_{4}, M_{f}^{2}\right)}{p_{T 4}} \\
& \times \frac{F_{i / H_{1}}\left(x_{1}, M^{2}\right)}{x_{1}} \frac{F_{j / H_{2}}\left(x_{2}, M^{2}\right)}{x_{2}}|M|_{3}^{2} \\
& +\int_{x_{4 m i n}}^{1} \frac{d x_{4}}{x_{4}} \int_{p_{T m}}^{p_{T 5 m a x}} d p_{T 5} p_{T 5} d \phi_{45}-C_{4} \\
& \times p_{T 4} D_{\gamma / l}\left(x_{4}, M_{f}^{2}\right) \frac{D_{\gamma / k}\left(x_{3}, M_{f}^{2}\right)}{p_{T 3}} \\
& \left.\times \frac{F_{i / H_{1}}\left(x_{1}, M^{2}\right)}{x_{1}} \frac{F_{j / H_{2}}\left(x_{2}, M^{2}\right)}{x_{2}}|M|_{4}^{2}\right]
\end{aligned}
$$

where

$$
\begin{aligned}
x_{1} & =\frac{p_{T 3}}{\sqrt{S}} e^{-y_{3}}+\frac{p_{T 4}}{\sqrt{S}} e^{-y_{4}}+\frac{p_{T 5}}{\sqrt{S}} e^{-y_{5}} \\
& =\hat{x}_{1}+\frac{p_{T 5}}{\sqrt{S}} e^{-y_{5}} \\
x_{2} & =\frac{p_{T 3}}{\sqrt{S}} e^{y_{3}}+\frac{p_{T 4}}{\sqrt{S}} e^{y_{4}}+\frac{p_{T 5}}{\sqrt{S}} e^{y_{5}} \\
& =\hat{x}_{2}+\frac{p_{T 5}}{\sqrt{S}} e^{y_{5}} \\
x_{3 \min } & =\frac{2 K_{T 3}}{\sqrt{S}} \cosh y_{3} \\
x_{4 \min } & =\frac{2 K_{T 4}}{\sqrt{S}} \cosh y_{4}
\end{aligned}
$$

The transverse momenta $p_{T 3}$ (resp. $p_{T 4}$ ) are the transverse momenta of the fragmenting partons. They are related to the photon variables by $p_{T 3}=K_{T 3} / x_{3}$ (resp. $p_{T 4}=K_{T 4} / x_{4}$ ). The integration range for the pair of variables $\phi_{35}$ (resp. $\phi_{45}$ ), $y_{5}$ is the kinematically allowed range minus a cone in rapidity azimuthal angle $C_{3}$ (resp. $C_{4}$ ) along the $\boldsymbol{p}_{3}$ (resp. $\boldsymbol{p}_{4}$ ) direction whose size is $R_{t h}$. The overall factor $C_{i j}$ reads:

$$
C_{i j}=\frac{\alpha_{s}^{3}\left(\mu^{2}\right)}{4 S^{2} \pi C_{i} C_{j}}
$$

and the $C_{i}$ are given by:

$$
C_{i}=\left\{\begin{array}{l}
N \text { for quarks } \\
\left(N^{2}-1\right) \text { for gluons }
\end{array}\right.
$$


The matrix element squared $[$, taken from the first reference of [0] and [34], has been split into two parts:

$$
|M|^{2}=|M|_{3}^{2}+|M|_{4}^{2}
$$

The first part $|M|_{3}^{2}$ contains final state collinear singularities arising when $\boldsymbol{p}_{3} / / \boldsymbol{p}_{5}$ and the second part $|M|_{4}^{2}$ contains final state collinear singularities arising when $\boldsymbol{p}_{4} / / \boldsymbol{p}_{5}$. More precisely, the matrix element squared can be written as a weighted sum of eikonal factors $E_{a b}$ plus a term free of infrared or collinear singularities:

$$
|M|_{i j \rightarrow k l m}^{2}=\sum_{a=1}^{4} \sum_{b=a+1}^{4} H_{a b}\left(p_{5}\right) E_{a b}+G\left(p_{5}\right)
$$

where

$$
E_{a b}=\frac{p_{a} \cdot p_{b}}{p_{a} \cdot p_{5} p_{b} \cdot p_{5}}
$$

Using:

$$
\frac{1}{p_{3} \cdot p_{5} p_{4} \cdot p_{5}}=\frac{1}{p_{1} \cdot p_{5}+p_{2} \cdot p_{5}}\left(\frac{1}{p_{3} \cdot p_{5}}+\frac{1}{p_{4} \cdot p_{5}}\right)
$$

we get:

$$
\begin{aligned}
|M|_{3}^{2} & =\frac{1}{2} H_{12}\left(p_{5}\right) E_{12}+H_{13}\left(p_{5}\right) E_{13}+H_{23}\left(p_{5}\right) E_{23}+H_{34}\left(p_{5}\right) E_{34}^{\prime}+\frac{1}{2} G\left(p_{5}\right) \\
|M|_{4}^{2} & =\frac{1}{2} H_{12}\left(p_{5}\right) E_{12}+H_{14}\left(p_{5}\right) E_{14}+H_{24}\left(p_{5}\right) E_{24}+H_{34}\left(p_{5}\right) E_{34}^{\prime \prime}+\frac{1}{2} G\left(p_{5}\right)
\end{aligned}
$$

with

$$
\begin{aligned}
& E_{34}^{\prime}=\frac{p_{3} \cdot p_{4}}{p_{1} \cdot p_{5}+p_{2} \cdot p_{5}} \frac{1}{p_{3} \cdot p_{5}} \\
& E_{34}^{\prime \prime}=\frac{p_{3} \cdot p_{4}}{p_{1} \cdot p_{5}+p_{2} \cdot p_{5}} \frac{1}{p_{4} \cdot p_{5}}
\end{aligned}
$$

In order that the infrared divergences cancel, and the collinear singularities factorize out, the coefficients $H_{a b}$ have to fulfill:

$$
\begin{aligned}
& H_{12}\left(\left(1-z_{1}\right) p_{1}\right)+H_{13}\left(\left(1-z_{1}\right) p_{1}\right)+H_{14}\left(\left(1-z_{1}\right) p_{1}\right)=\frac{1}{z_{1}}\left[a_{i^{\prime} i}^{(d)}\left(z_{1}\right) \frac{C_{i}}{C_{i^{\prime}}}|M|_{i^{\prime} j \rightarrow k l}^{2 B}\right] \\
& H_{12}\left(\left(1-z_{2}\right) p_{2}\right)+H_{23}\left(\left(1-z_{2}\right) p_{2}\right)+H_{24}\left(\left(1-z_{2}\right) p_{2}\right)=\frac{1}{z_{2}}\left[a_{j^{\prime} j}^{(d)}\left(z_{2}\right) \frac{C_{j}}{C_{j^{\prime}}}|M|_{i j^{\prime} \rightarrow k l}^{2 B}\right] \\
& H_{13}\left(\frac{1-z_{3}}{z_{3}} p_{3}\right)+H_{23}\left(\frac{1-z_{3}}{z_{3}} p_{3}\right)+H_{34}\left(\frac{1-z_{3}}{z_{3}} p_{3}\right)=\frac{1}{z_{3}}\left[a_{k k^{\prime}}^{(d)}\left(z_{3}\right)|M|_{i j \rightarrow k^{\prime} l}^{2 B}\right] \\
& H_{14}\left(\frac{1-z_{4}}{z_{4}} p_{4}\right)+H_{24}\left(\frac{1-z_{4}}{z_{4}} p_{4}\right)+H_{34}\left(\frac{1-z_{4}}{z_{4}} p_{4}\right)=\frac{1}{z_{4}}\left[a_{l l^{\prime}}^{(n)}\left(z_{4}\right)|M|_{i j \rightarrow k l^{\prime}}^{2 B}\right]
\end{aligned}
$$

In particular, the cancellation of infrared divergences is insured by:

$$
\begin{aligned}
H_{12}(0)+H_{13}(0)+H_{14}(0) & =a_{i i}^{(d)}(1)|M|_{i j \rightarrow k l}^{2 B} \\
H_{12}(0)+H_{23}(0)+H_{24}(0) & =a_{j j}^{(d)}(1)|M|_{i j \rightarrow k l}^{2 B} \\
H_{13}(0)+H_{23}(0)+H_{34}(0) & =a_{k k}^{(d)}(1)|M|_{i j \rightarrow k l}^{2 B} \\
H_{14}(0)+H_{24}(0)+H_{34}(0) & =a_{l l}^{(d)}(1)|M|_{i j \rightarrow k l}^{2 B} .
\end{aligned}
$$

\footnotetext{
${ }^{11}$ An overall factor of the matrix element squared containing the average on spins and colours of the initial state and the coupling constant has been put into the coefficient $C_{i j}$
} 
The functions $a_{i j}^{(d)}(z)$ will be given in equation (A.13).

In equation (A.1), the integration domain for the rapidities and the transverse momenta of the two photons is in general limited by experiments. The integration over $p_{T 5}$ is constrained by:

$$
p_{T 5}^{2}<S\left(1-\hat{x}_{1}\right)\left(1-\hat{x}_{2}\right)
$$

\section{A.2 Pseudo cross sections for the initial state collinear parts}

The finite part associated to the collinear divergence $\boldsymbol{p}_{1} / / \boldsymbol{p}_{5}$ is given by:

$$
\begin{aligned}
\sigma_{5 / / 1}= & \int d y_{3} \int d y_{4} \int d K_{T 3} \int d K_{T 4} \\
& \times \frac{\alpha_{s}\left(\mu^{2}\right)}{2 \pi} C_{i j}^{B} \int_{x_{3 m i n}}^{1} \frac{d x_{3}}{x_{3}} \int_{x_{4 m i n}}^{1} \frac{d x_{4}}{x_{4}} p_{T} \delta\left(p_{T 3}-p_{T 4}\right) \\
& \times D_{\gamma / k}\left(x_{3}, M_{f}^{2}\right) D_{\gamma / l}\left(x_{4}, M_{f}^{2}\right) \int_{x_{1}^{0}}^{1} \frac{d z_{1}}{z_{1}} \frac{F_{i / H_{1}}\left(\frac{x_{1}^{0}}{z_{1}}, M^{2}\right)}{x_{1}^{0}} \frac{F_{j / H_{2}}\left(x_{2}^{0}, M^{2}\right)}{x_{2}^{0}} \\
& \times \frac{C_{i}}{C_{i^{\prime}}}\left[\left(\frac{a_{i^{\prime} i}^{(d-4)}\left(z_{1}\right)}{\left(1-z_{1}\right)_{+}}+\ln \left(\frac{p_{T m}^{2}}{M^{2}}\right) P_{i^{\prime} i}^{(4)}\left(z_{1}\right)-f_{i^{\prime} i}\left(z_{1}\right)\right)\right]|M|_{i^{\prime} j \rightarrow k l}^{2 B}
\end{aligned}
$$

where the variables $x_{1}^{0}$ (resp. $x_{2}^{0}$ ) are defined by:

$$
\begin{aligned}
& x_{1}^{0}=\frac{p_{T}}{\sqrt{S}}\left(e^{-y_{3}}+e^{-y_{4}}\right) \\
& x_{2}^{0}=\frac{p_{T}}{\sqrt{S}}\left(e^{y_{3}}+e^{y_{4}}\right)
\end{aligned}
$$

and $p_{T}$ stands for $p_{T 3}$ or $p_{T 4}$.

The finite part associated to the collinear divergence $\boldsymbol{p}_{2} / / \boldsymbol{p}_{5}$ is given by:

$$
\begin{aligned}
\sigma_{5 / / 2}= & \int d y_{3} \int d y_{4} \int d K_{T 3} \int d K_{T 4} \\
& \times \frac{\alpha_{s}\left(\mu^{2}\right)}{2 \pi} C_{i j}^{B} \int_{x_{3 m i n}}^{1} \frac{d x_{3}}{x_{3}} \int_{x_{4 m i n}}^{1} \frac{d x_{4}}{x_{4}} p_{T} \delta\left(p_{T 3}-p_{T 4}\right) \\
& \times D_{\gamma / k}\left(x_{3}, M_{f}^{2}\right) D_{\gamma / l}\left(x_{4}, M_{f}^{2}\right) \int_{x_{2}^{0}}^{1} \frac{d z_{2}^{0}}{z_{2}} \frac{F_{i / H_{1}}\left(x_{1}^{0}, M^{2}\right)}{x_{1}^{0}} \frac{F_{j / H_{2}}\left(\frac{x_{2}^{0}}{z_{2}}, M^{2}\right)}{x_{2}^{0}} \\
& \times \frac{C_{j}}{C_{j^{\prime}}}\left[\left(\frac{a_{j^{\prime} j}^{(d-4)}\left(z_{2}\right)}{\left(1-z_{2}\right)_{+}}+\ln \left(\frac{p_{T m}^{2}}{M^{2}}\right) P_{j^{\prime} j}^{(4)}\left(z_{2}\right)-f_{j^{\prime} j}\left(z_{2}^{0}\right)\right)\right]|M|_{i j^{\prime} \rightarrow k l}^{2 B}
\end{aligned}
$$

with

$$
C_{i j}^{B}=\frac{2 \pi \alpha_{s}^{2}\left(\mu^{2}\right)}{4 S^{2} C_{i} C_{j}}
$$

The functions $a_{i j}^{(d-4)}(z), P_{i j}^{(4)}(z)$ and $f_{i j}(z)$ will be defined at the end of this appendix cf. equations from (A.18) to (A.21), (A.13) and (A.24).

\section{A.3 Pseudo cross section for the final state collinear parts}

These parts contain the collinear singularities which have been absorbed into the bare fragmentation functions. 
The finite part associated to the collinear divergence $\boldsymbol{p}_{3} / / \boldsymbol{p}_{5}$ is given by:

$$
\begin{aligned}
\sigma_{5 / / 3}= & \int d y_{3} \int d y_{4} \int d K_{T 3} \int d K_{T 4} \\
& \times \frac{\alpha_{s}\left(\mu^{2}\right)}{2 \pi} C_{i j}^{B} \int_{x_{3 m i n}}^{1} \frac{d x_{3}}{x_{3}} \int_{z_{3 \min }}^{1} \frac{d z_{3}}{z_{3}} \\
& \times D_{\gamma / k}\left(x_{3}, M_{f}^{2}\right) D_{\gamma / l}\left(x_{4}^{\prime}, M_{f}^{2}\right) \frac{F_{i / H_{1}}\left(x_{1}^{\prime}, M^{2}\right)}{x_{1}^{\prime}} \frac{F_{j / H_{2}}\left(x_{2}^{\prime}, M^{2}\right)}{x_{2}^{\prime}} \\
& \times\left[\left(\frac{a_{k k^{\prime}}^{(d-4)}\left(z_{3}\right)}{\left(1-z_{3}\right)_{+}}+\ln \left(\frac{p_{T 3}^{2}}{M_{f}^{2}}\right) P_{k k^{\prime}}^{(4)}\left(z_{3}\right)-d_{k k^{\prime}}\left(z_{3}\right)+2\left(\frac{\ln \left(1-z_{3}\right)}{\left(1-z_{3}\right)}\right)_{+} a_{k k^{\prime}}^{(4)}\left(z_{3}\right)\right.\right. \\
& \left.\left.+\ln \left(R^{2}\right) \frac{a_{k k^{\prime}}^{(4)}\left(z_{3}\right)}{\left(1-z_{3}\right)} \theta\left(z_{3 m}-z_{3}\right)\right)\right]|M|_{i j \rightarrow k^{\prime} l}^{2 B}
\end{aligned}
$$

wheras the finite part associated to the collinear divergence $\boldsymbol{p}_{4} / / \boldsymbol{p}_{5}$ is given by:

$$
\begin{aligned}
\sigma_{5 / / 4}= & \int d y_{3} \int d y_{4} \int d K_{T 3} \int d K_{T 4} \\
& \times \frac{\alpha_{s}\left(\mu^{2}\right)}{2 \pi} C_{i j}^{B} \int_{x_{4 m i n}}^{1} \frac{d x_{4}}{x_{4}} \int_{z_{4 m i n}}^{1} \frac{d z_{4}}{z_{4}} \\
& \times D_{\gamma / k}\left(x_{3}^{\prime \prime}, M_{f}^{2}\right) D_{\gamma / l}\left(x_{4}, M_{f}^{2}\right) \frac{F_{i / H_{1}}\left(x_{1}^{\prime \prime}, M^{2}\right)}{x_{1}^{\prime \prime}} \frac{F_{j / H_{2}}\left(x_{2}^{\prime \prime}, M^{2}\right)}{x_{2}^{\prime \prime}} \\
& \times\left[\left(\frac{a_{l l^{\prime}}^{(d-4)}\left(z_{4}\right)}{\left(1-z_{4}\right)_{+}}+\ln \left(\frac{p_{T 4}^{2}}{M_{f}^{2}}\right) P_{l l^{\prime}}^{(4)}\left(z_{4}\right)-d_{l l^{\prime}}\left(z_{4}\right)+2\left(\frac{\ln \left(1-z_{4}\right)}{\left(1-z_{4}\right)}\right)_{+} a_{l l^{\prime}}^{(4)}\left(z_{4}\right)\right.\right. \\
& \left.\left.+\ln \left(R^{2}\right) \frac{a_{l l^{\prime}}^{(4)}\left(z_{4}\right)}{\left(1-z_{4}\right)} \theta\left(z_{4 m}-z_{4}\right)\right)\right]|M|_{i j \rightarrow k l^{\prime}}^{2 B}
\end{aligned}
$$

The functions $a_{i j}^{(4)}(z)$ and $d_{i j}(z)$ will be also defined at the end of the appendix cf. equations from (A.14) to (A.17) and (A.25). The variables $z_{3 m}, z_{4 m}, x_{1}^{\prime}, x_{2}^{\prime}, x_{1}^{\prime \prime}$ and $x_{2}^{\prime \prime}$ appearing in equations (A.10) and (A.11) are given by:

$$
\begin{aligned}
z_{3 m} & =\frac{p_{T 3}}{p_{T 3}+p_{T m}} \\
z_{4 m} & =\frac{p_{T 4}}{p_{T 4}+p_{T m}} \\
x_{1}^{\prime} & =\frac{p_{T 3}+p_{T 5}}{\sqrt{S}}\left(e^{y_{3}}+e^{y_{4}}\right) \\
x_{2}^{\prime} & =\frac{p_{T 3}+p_{T 5}}{\sqrt{S}}\left(e^{-y_{3}}+e^{-y_{4}}\right) \\
x_{1}^{\prime \prime} & =\frac{p_{T 4}+p_{T 5}}{\sqrt{S}}\left(e^{y_{3}}+e^{y_{4}}\right) \\
x_{2}^{\prime \prime} & =\frac{p_{T 4}+p_{T 5}}{\sqrt{S}}\left(e^{-y_{3}}+e^{-y_{4}}\right)
\end{aligned}
$$

\section{A.4 Pseudo cross section for the infrared and virtual parts}

This pseudo cross section is given by:

$$
\begin{aligned}
\sigma_{i r}= & \int d y_{3} \int d y_{4} \int d K_{T 3} \int d K_{T 4} \\
& \times \frac{\alpha_{s}\left(\mu^{2}\right)}{2 \pi} C_{i j}^{B} \int_{x_{0}}^{1} \frac{d x_{3}}{x_{3}} \int_{x_{4}}^{1} \frac{d x_{4}}{x_{4}} p_{T} \delta\left(p_{T 3}-p_{T 4}\right)
\end{aligned}
$$




$$
\begin{aligned}
& \times D_{\gamma / k}\left(x_{3}, M_{f}^{2}\right) D_{\gamma / l}\left(x_{4}, M_{f}^{2}\right) \frac{F_{i / H_{1}}\left(x_{1}^{0}, M^{2}\right)}{x_{1}^{0}} \frac{F_{j / H_{2}}\left(x_{2}^{0}, M^{2}\right)}{x_{2}^{0}} \\
& \times\left\{-\left[\ln \left(\frac{p_{T}^{2}}{S}\right)\left(b_{k k}+b_{l l}\right)+\ln \left(\frac{p_{T m}^{2}}{S}\right)\left(b_{i i}+b_{j j}\right)\right]|M|_{i j \rightarrow k l}^{2 B}\right. \\
& +\ln \left(\frac{p_{T m}^{2}}{S}\right)\left(\sum_{i<j} H_{i j}(0) \ln \left(\frac{2 p_{i}^{0} \cdot p_{j}^{0}}{S}\right)\right) \\
& -\frac{1}{2} \ln \left(\frac{p_{T}^{2}}{S}\right) \ln \left(\frac{p_{T m}^{2}}{S}\right)\left[H_{13}(0)+H_{14}(0)+H_{23}(0)+H_{24}(0)+2 H_{34}(0)\right] \\
& +\frac{1}{4} \ln ^{2}\left(\frac{p_{T}^{2}}{S}\right)\left[H_{13}(0)+H_{14}(0)+H_{23}(0)+H_{24}(0)+2 H_{34}(0)\right] \\
& -\frac{1}{4} \ln ^{2}\left(\frac{p_{T m}^{2}}{S}\right)\left[2 H_{12}(0)+H_{13}(0)+H_{14}(0)+H_{23}(0)+H_{24}(0)\right] \\
& \left.+\frac{H_{34}(0)}{\pi}\left[A_{34}\left(y^{\star}\right)+A_{34}\left(-y^{\star}\right)\right]+F(\hat{s}, \hat{t}, \hat{u})\right\}
\end{aligned}
$$

with $p_{T}=p_{T 3}=p_{T 4}$. The terms $b_{i i}$ are defined in equations (A.22) and (A.23). In the equation (A.12), $y^{\star}=\left(y_{3}-y_{4}\right) / 2$ and the function $A(x)$ is given by:

$$
\begin{aligned}
A(x)= & \pi \ln (2) \ln \left(4 \cosh ^{2}\left(y^{\star}\right)\right)+2 y^{\star} \sinh \left(2 y^{\star}\right) \int_{0}^{\pi} d \phi \frac{\ln (\sin \phi)}{\cosh (2 x)+\cos (2 \phi)} \\
& +4 \int_{0}^{\pi} d \phi \frac{\sin (2 \phi)}{\cosh (2 x)+\cos (2 \phi)} \ln (\sin \phi) \arctan \left(\frac{\sin \phi}{1-\cos \phi}\right)
\end{aligned}
$$

The function $F$ is the finite part of the virtual term and the variables $\hat{s}, \hat{t}$ and $\hat{u}$ are the Mandelstam variables of the $2 \rightarrow 2$ processes:

$$
\begin{aligned}
\hat{s} & =\left(p_{1}^{0}+p_{2}^{0}\right)^{2} \\
\hat{t} & =\left(p_{1}^{0}-p_{3}^{0}\right)^{2} \\
\hat{u} & =\left(p_{2}^{0}-p_{3}^{0}\right)^{2}
\end{aligned}
$$

where the 4 -vectors $p_{i}^{0}$ are the infrared limits of the 4 -vectors $p_{i}$.

\section{A.5 Altarelli-Parisi Kernels}

We will give in this appendix the expressions of the functions $a_{i j}(z)$ and $b_{i j}$. These functions are defined by:

$$
\begin{aligned}
P_{i j}^{(d)}(z) & =\frac{a_{i j}^{(d)}(z)}{(1-z)_{+}}+b_{i j} \delta(1-z) \\
& =\frac{a_{i j}^{(4)}(z)-\epsilon a_{i j}^{(d-4)}(z)}{(1-z)_{+}}+b_{i j} \delta(1-z) \\
& =P_{i j}^{(4)}(z)-\epsilon \frac{a_{i j}^{(d-4)}(z)}{(1-z)_{+}}
\end{aligned}
$$

where $P_{i j}^{(4)}$ (resp. $P_{i j}^{(d)}$ ) are the Altarelli-Parisi Kernels in four (resp. $d$ ) dimensions. So the expressions for the functions $a_{i j}^{(4)}(z), a_{i j}^{(d-4)}(z)$ and $b_{i j}$ are given by:

$$
a_{g g}^{(4)}(z)=2 N\left(z+\frac{(1-z)^{2}}{z}+z(1-z)^{2}\right)
$$




$$
\begin{aligned}
& a_{q q}^{(4)}(z)=C_{F}\left(1+z^{2}\right) \\
& a_{g q}^{(4)}(z)=C_{F}\left(\frac{1+(1-z)^{2}}{z}\right)(1-z) \\
& a_{q g}^{(4)}(z)=T_{F}\left(z^{2}+(1-z)^{2}\right)(1-z)
\end{aligned}
$$

where $N$ is the number of colors, $C_{F}=\left(N^{2}-1\right) /(2 N)$ and $T_{F}=1 / 2$. The extra part needed to get the functions $a$ in $d$ dimensions $\left(a_{i j}^{(d)}(z)=a_{i j}^{(4)}(z)-\epsilon a_{i j}^{(d-4)}(z)\right)$ is given by:

$$
\begin{aligned}
a_{g g}^{(d-4)}(z) & =0 \\
a_{q q}^{(d-4)}(z) & =C_{F}(1-z)^{2} \\
a_{g q}^{(d-4)}(z) & =C_{F} z(1-z) \\
a_{q g}^{(d-4)}(z) & =2 T_{F} z(1-z)^{2}
\end{aligned}
$$

The coefficients $b_{i j}$ read:

$$
\begin{aligned}
b_{g g} & =\frac{\left(11 N-2 N_{F}\right)}{6} \\
b_{q q} & =\frac{3}{2} C_{F}
\end{aligned}
$$

The function $f_{i j}(x)$ and $d_{i j}(z)$ define the factorisation scheme for respectively initial state and final state collinear singularities. In the $\overline{M S}$ scheme, we have:

$$
\begin{aligned}
& f_{i j}(z)=0 \\
& d_{i j}(z)=0
\end{aligned}
$$

\section{References}

[1] WA70 Collaboration, E. Bonvin et al., Z. Phys. C41 (1989) 591.

[2] WA70 Collaboration, E. Bonvin et al., Phys. Lett. 236B (1990) 523.

[3] Private communication from M. Begel (E706 collaboration).

[4] UA2 Collaboration. J. Alitti et al., Phys. Lett. 288B (1992) 386.

[5] CDF Collaboration, F. Abe et al., Phys. Rev. Lett. 70 (1993) 2232.

[6] Wei Chen, PhD Thesis (Univ. New-York at Stony Brook), Dec. 1997, unpublished;

D0 Collaboration (P. Hanlet for the collaboration), Nucl. Phys. Proc. Suppl. 64 (1998) 78.

All the numbers used in this article are taken from tables in Wei Chen PhD Thesis.

[7] P. Aurenche, R. Baier, A. Douiri, M. Fontannaz and D. Schiff, Z. Phys. C29 (1985) 459;

P. Aurenche, M. Bonesini, L. Camilleri, M. Fontannaz and M. Werlen, Proceedings of LHC Aachen Workshop CERN-90-10 G. Jarlskog and D. Rein eds., vol. II, p. 83.

[8] B. Bailey, J. Ohnemus and J.F. Owens, Phys. Rev. D46 (1992) 2018;

B. Bailey and J.F. Owens, Phys. Rev. D47 (1993) 2735;

B. Bailey and D. Graudenz, Phys. Rev. D49 (1994) 1486.

[9] C. Balazs, E.L. Berger, S. Mrenna and C.P. Yuan, Phys. Rev. D57 (1998) 6934;

C. Balazs and C.P. Yuan, Phys. Rev. D59 (1999) 114007.

[10] L. Bourhis, M. Fontannaz and J.Ph. Guillet, Eur. Phys. J. C2 (1998) 529. 
[11] A. Djouadi, D. Graudenz, M. Spira and P. Zerwas, Nucl. Phys. B453 (1995) 17.

[12] V. Del Duca, W.B. Kilgore and F. Maltoni, hep-ph/9910253.

[13] D. de Florian and Z. Kunszt, Phys. Lett. 460B (1999) 184;

C. Balazs, P. Nadolsky, C. Schmidt and C.P. Yuan, hep-ph/9905551.

[14] V.A. Smirnov, Phys. Lett. 460B (1999) 397;

J.B. Tausk, hep-ph/9909506.

[15] P. Chiappetta, R. Fergani and J.-Ph Guillet, Z. Phys. C69 (1996) 443.

[16] M.A. Furman Nucl. Phys. B197 (1982) 413;

W.T. Giele, E.W.N. Glover and D. Kosower, Nucl. Phys. B403 (1993) 633;

see also the first article of [8].

[17] R.K. Ellis, D.A. Ross and A.E. Terrano, Nucl. Phys. B187 (1981) 421;

Z. Kunszt and D.E. Soper, Phys. Rev. D46 (1992) 192.

Concerning the dipole formalism, see:

S. Catani and M.H. Seymour, Nucl. Phys. B485 (1997) 291.

[18] S. Kawabata, Comp. Phys. Comm. 88 (1995) 309.

[19] R. Brun, O. Couet, G.E. Vandoni and P. Zabarini, Comp. Phys. Comm 57 (1989) 432;

PAW, Physics Analysis Workstation, CERN Program Library Q121

(http://wwwinfo.cern.ch/asd/paw/index.html).

[20] H.-U. Bengtsson and T. Sjöstrand, Comp. Phys. Comm. 46 (1987) 43;

T. Sjöstrand, Comp. Phys. Comm. 82 (1994) 74;

T. Sjöstrand, LU TP 95-20, hep-ph/9508391.

[21] G. Marchesini and B.R. Webber, Nucl. Phys. B 310 (1988) 461;

G. Marchesini, B.R. Webber, G. Abbiendi, I.G. Knowles, M.H. Seymour and L. Stanco, hep-ph/9607393.

[22] P. Aurenche, R. Baier, M. Fontannaz, J. Owens and M. Werlen, Phys. Rev. D39 (1989) 3275.

[23] A.D. Martin, R.G. Roberts and W.J. Stirling, Phys. Lett. 354B (1995) 155.

[24] CMS technical proposal CERN/LHCC 94-38 (p 179);

ATLAS technical proposal CERN/LHCC 94-43;

V. Tisserand for the ATLAS collaboration, in Proc. 6th Int. Conf. on Calorimetry in High Energy Physics (ICCHEP 96), ed. by A. Antonelli, S. Bianco, A. Calcaterra, F.L. Fabbri, (Frascati physics series; 6) p 475.

[25] A.D. Martin, R.G. Roberts, W.J. Stirling and R.S. Thorne, hep-ph/9907231.

[26] Y. Dokshitzer, D. Dyakonov and S. Troyan, Phys. Rep. 58 (1980) 269;

A. Basseto, M. Ciafaloni and G. Marchesini, Phys. Rep. 100 (1983) 201 and references therein; see also [28].

[27] C. Balazs, private communication.

[28] J. Collins and D. Soper, Nucl. Phys. B193 (1981) 381;

J. Collins and D. Soper, Nucl. Phys. B197 (1982) 446;

J. Collins, D. Soper and G. Sterman, Nucl. Phys. B250 (1985) 199. 
[29] R. Bonciani, S. Catani, M. Mangano and P. Nason, Nucl. Phys. B529 (1998) 424;

N. Kidonakis, G. Oderda and G. Sterman, Nucl. Phys. B531 (1998) 365.

[30] E.L. Berger, X.F. Guo and J.W. Qiu, Phys. Rev D54 (1996) 5470.

[31] S. Catani, M. Fontannaz and E. Pilon, Phys. Rev. D58 (1998) 094025.

[32] S. Catani and B. Webber, JHEP 9710:005 (1997).

[33] S. Frixione, Phys. Lett. 429B (1998) 369.

[34] R. K. Ellis and J. C. Sexton, Nucl. Phys. B269 (1986) 445.

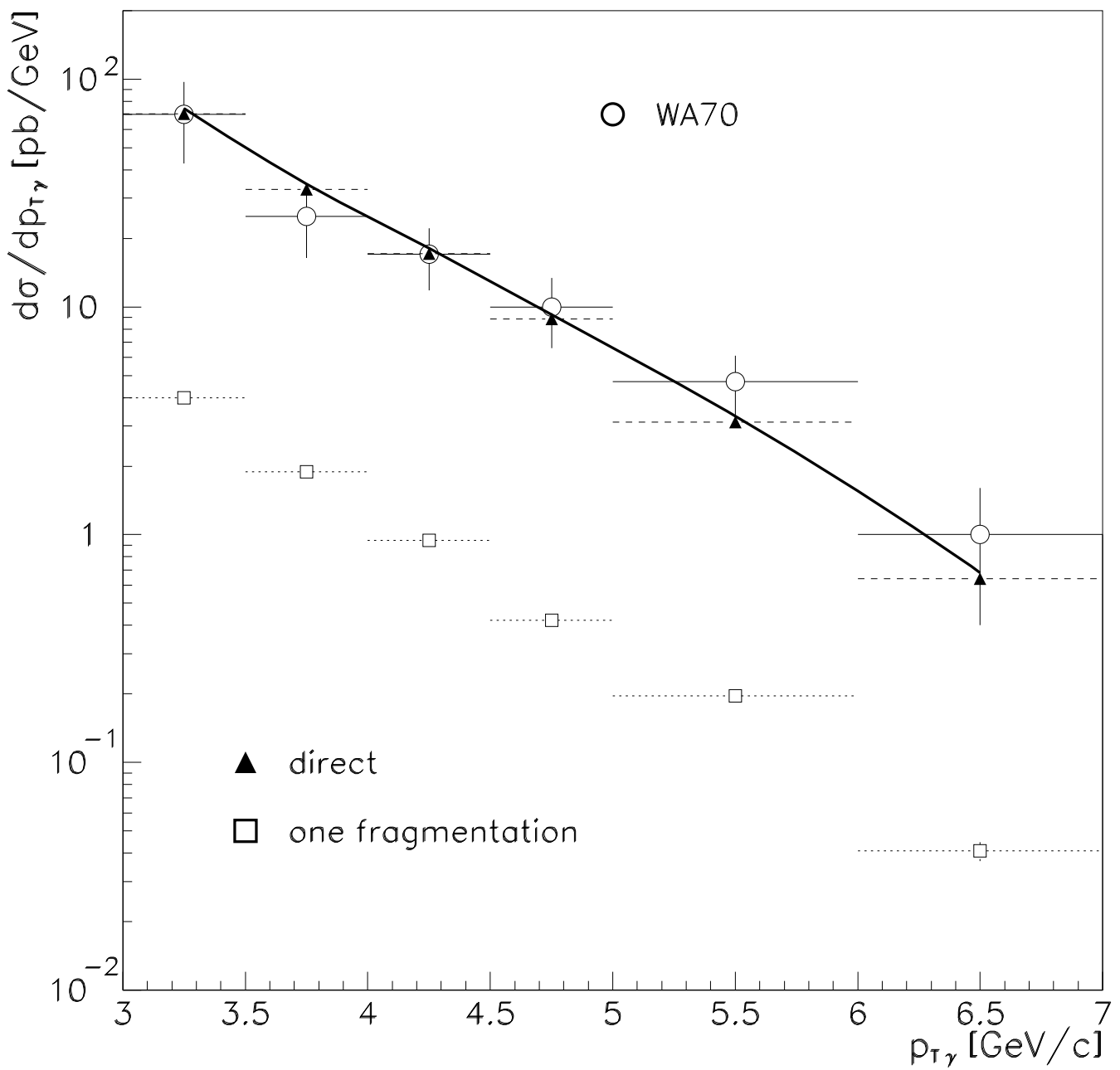

Figure 1: Diphoton differential cross section $d \sigma / d p_{T}$ vs. $p_{T}$, the transverse energy of each photon, in $\pi^{-}$-proton collisions at $\sqrt{S}=22.9 \mathrm{GeV}$. Data points from the WA70 collaboration [1]. The solid line is the full contribution with scales $M=\mu=M_{f}=0.275\left(p_{T}\left(\gamma_{1}\right)+p_{T}\left(\gamma_{2}\right)\right)$. 

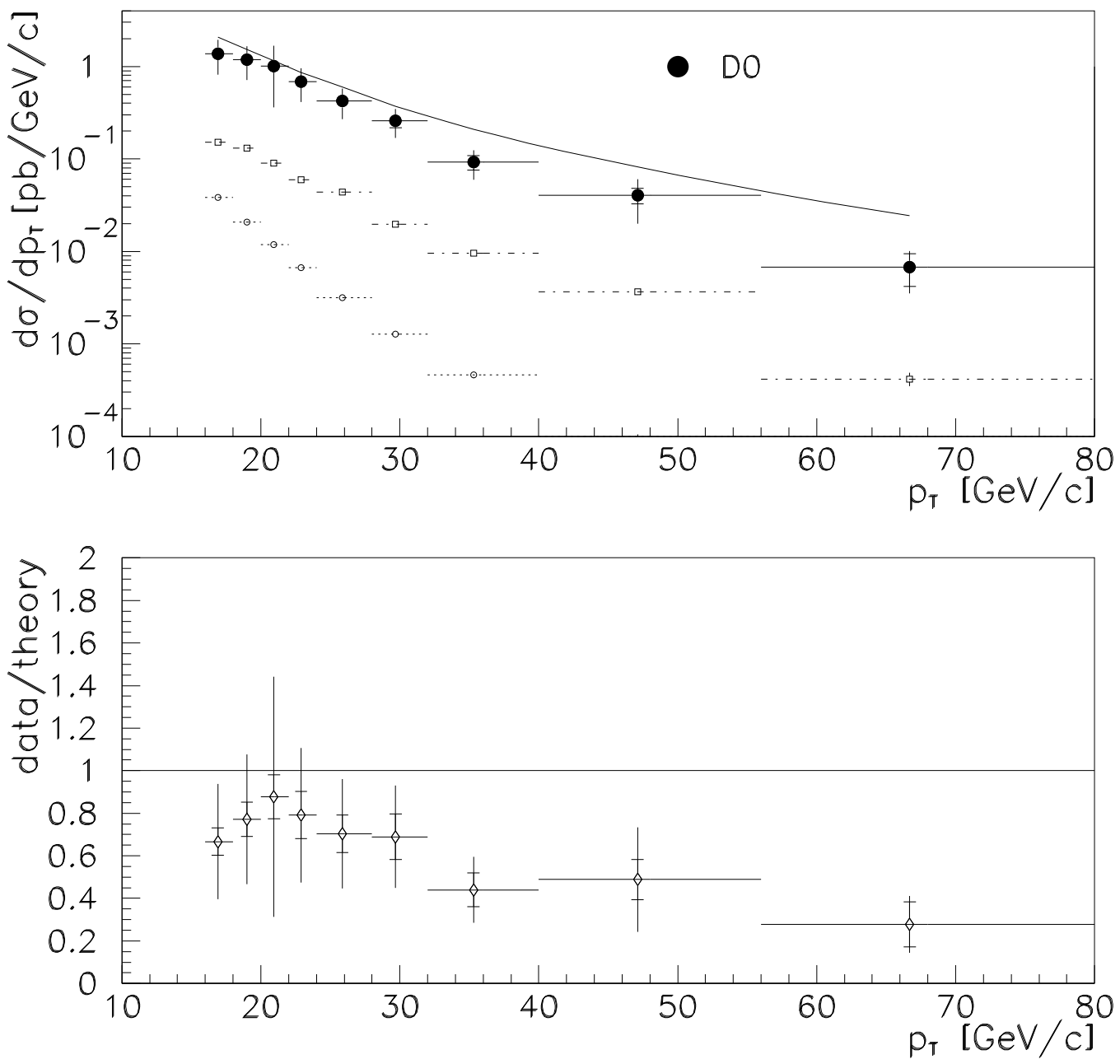

Figure 2: Diphoton differential cross section $d \sigma / d p_{T}$ vs $p_{T}$, the transverse energy of each photon, at Tevatron, $\sqrt{S}=1.8 \mathrm{TeV}$. Preliminary data points (statistical errors and systematics in quadrature) from the D0 collaboration [6] are compared to the theoretical predictions: the full NLO prediction is shown as the solid line while open squares (open circles) represent the single (double) fragmentation contribution. The ratio data/(full NLO theory) is shown below. 

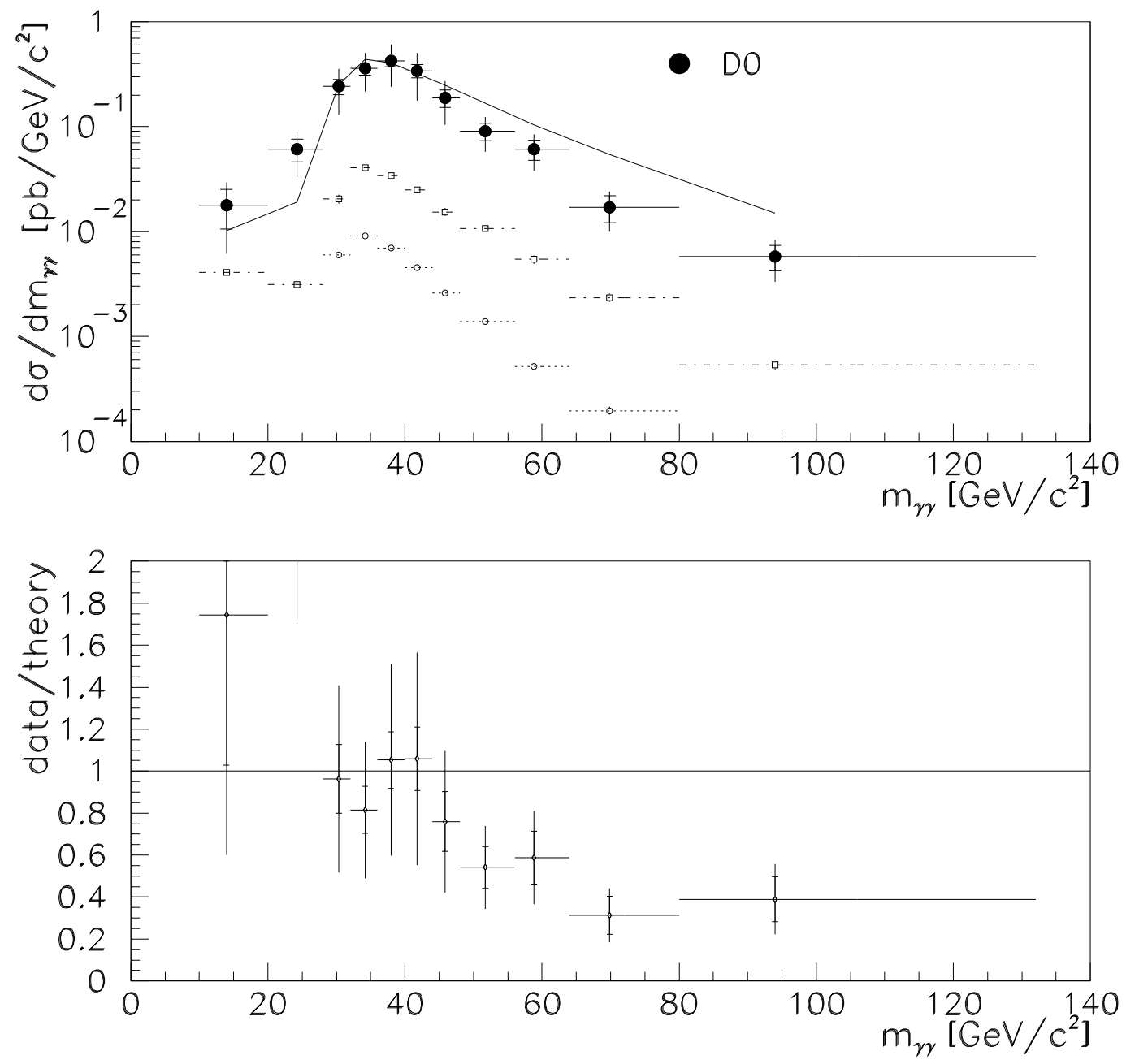

Figure 3: Diphoton differential cross section $d \sigma / d m_{\gamma \gamma}$ vs. $m_{\gamma \gamma}$, the mass of the photon pair, at Tevatron, $\sqrt{S}=1.8 \mathrm{TeV}$. Preliminary data points (statistical errors and systematics in quadrature) from the D0 collaboration [6] are compared to the theoretical predictions: the full NLO prediction is shown as the solid line while open squares (open circles) represent the single (double) fragmentation contribution. 

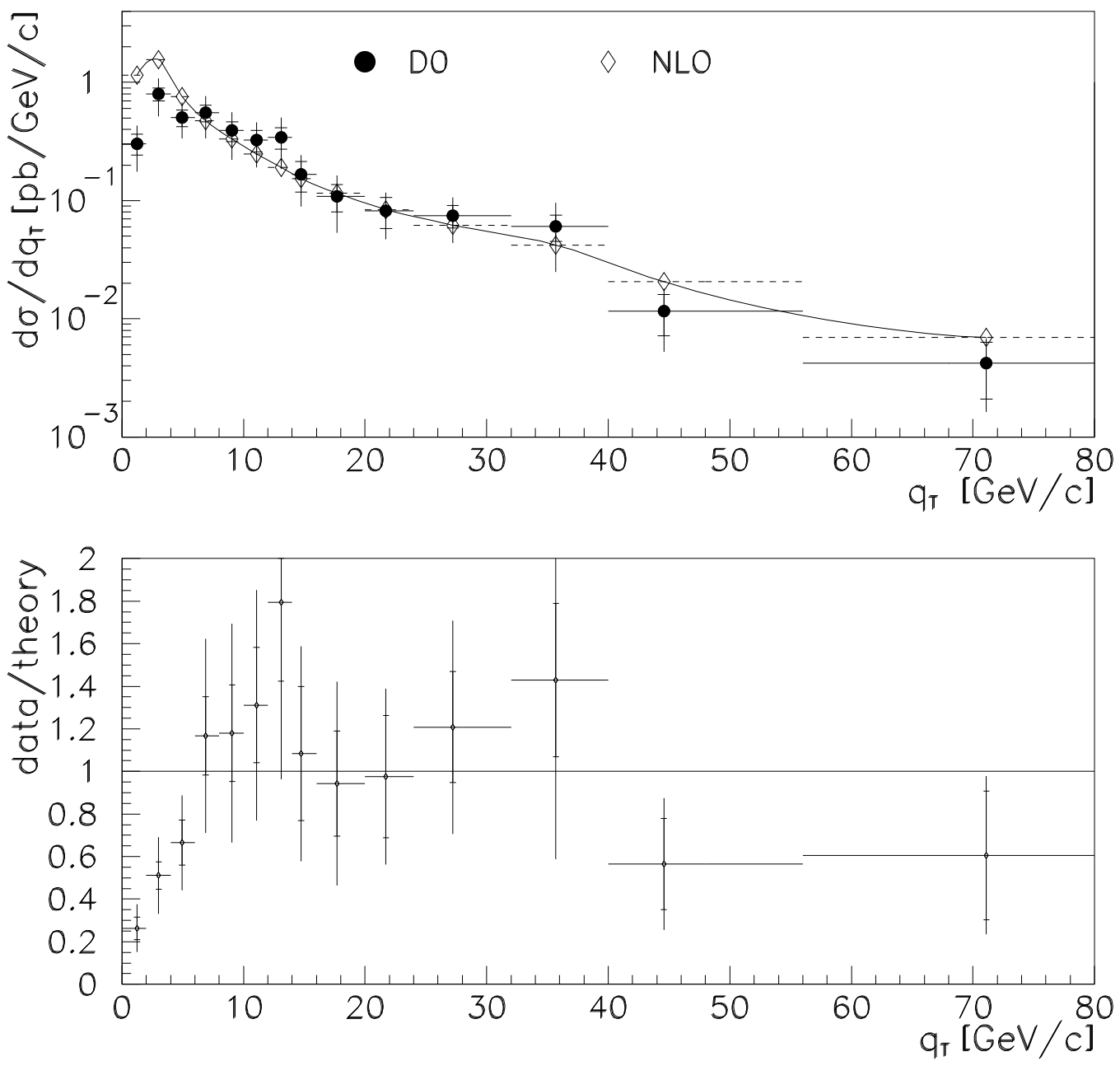

Figure 4: Diphoton differential cross section $d \sigma / d q_{T}$ vs. $q_{T}$, the transverse momentum of the photon pair, at Tevatron, $\sqrt{S}=1.8 \mathrm{TeV}$. Preliminary data points (statistical errors and systematics in quadrature) from the D0 collaboration [6] are compared to the theoretical predictions: the full NLO prediction is shown as the solid line 

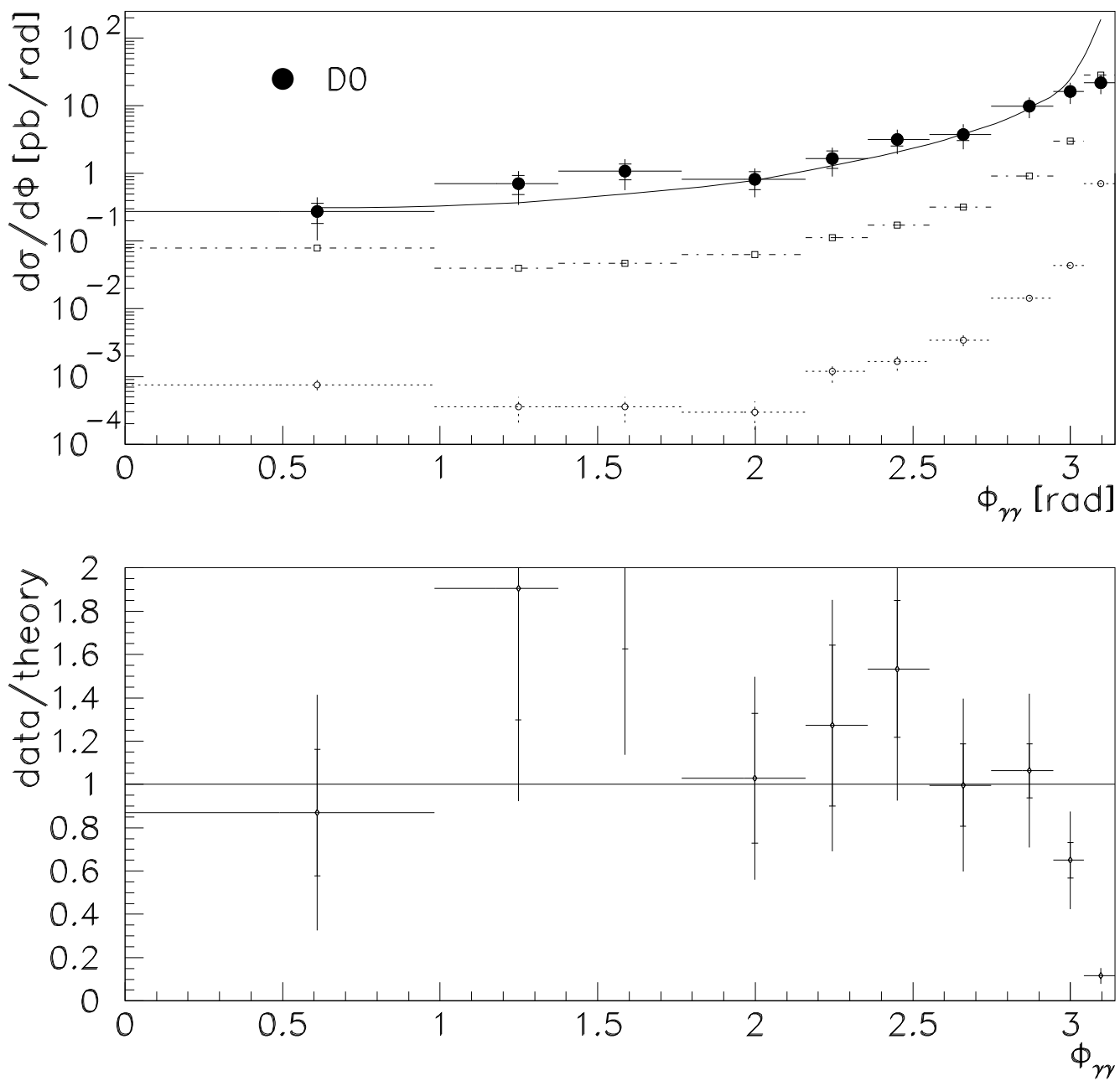

Figure 5: Diphoton differential cross section $d \sigma / d \phi_{\gamma \gamma}$ vs. $\phi_{\gamma \gamma}$, the azimuthal angle between the two photons, at Tevatron, $\sqrt{S}=1.8 \mathrm{TeV}$. Preliminary data points (statistical errors and systematics in quadrature) from the D0 collaboration [6] are compared to the theoretical predictions: the full NLO prediction is shown as the solid line while open squares (open circles) represent the single (double) fragmentation contribution. 

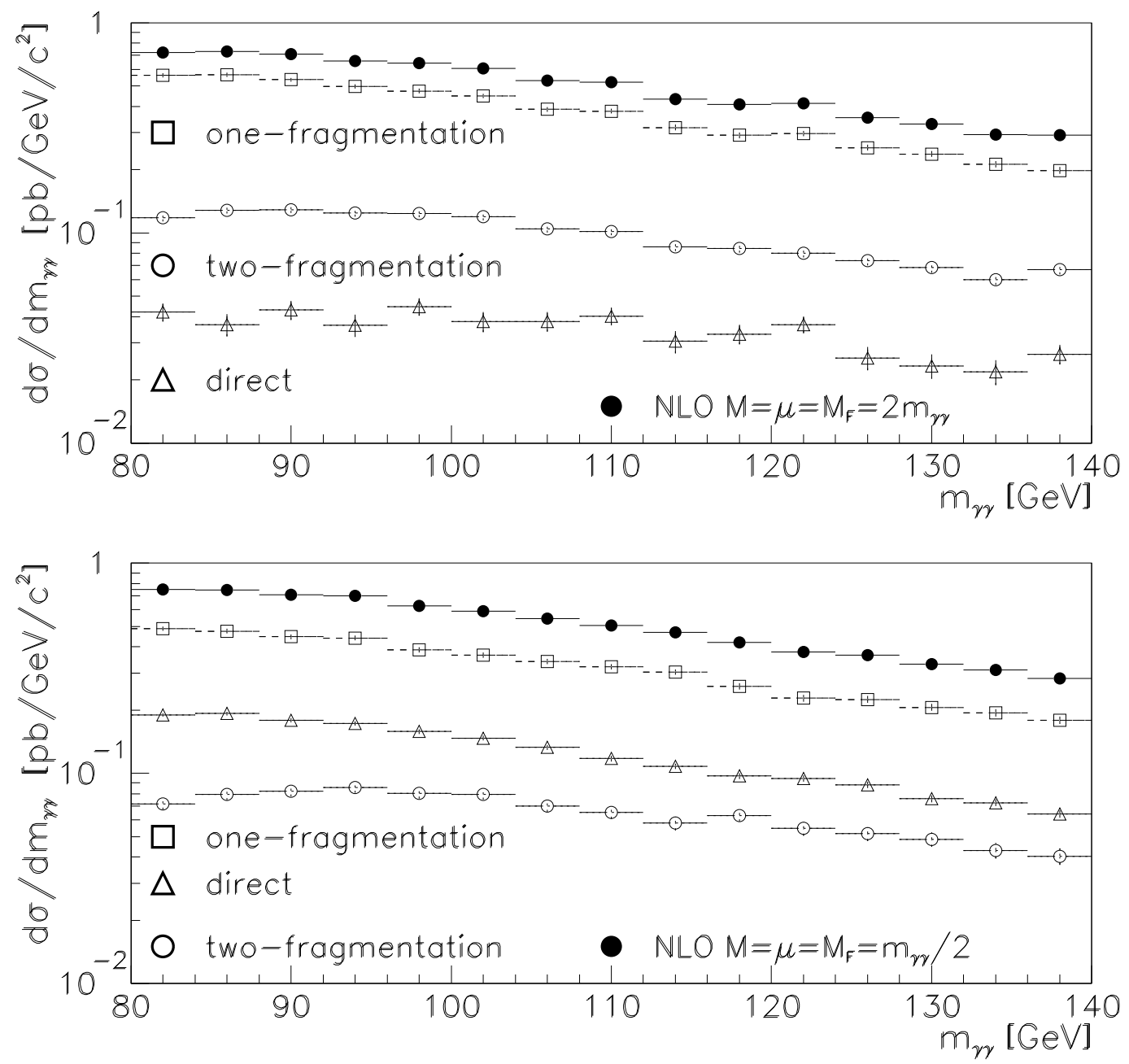

Figure 6: Splitting of the diphoton differential cross section $d \sigma / d m_{\gamma \gamma}$ at LHC, $\sqrt{S}=14 \mathrm{TeV}$ without isolation, into the "direct", "one fragmentation" and "two fragmentation" components, shown for two different choices of scales. The following kinematic cuts are applied: $p_{T}\left(\gamma_{1}\right) \geq 40 \mathrm{GeV}, p_{T}\left(\gamma_{2}\right) \geq 25$ $\mathrm{GeV},\left|y\left(\gamma_{1,2}\right)\right| \leq 2.5$. 

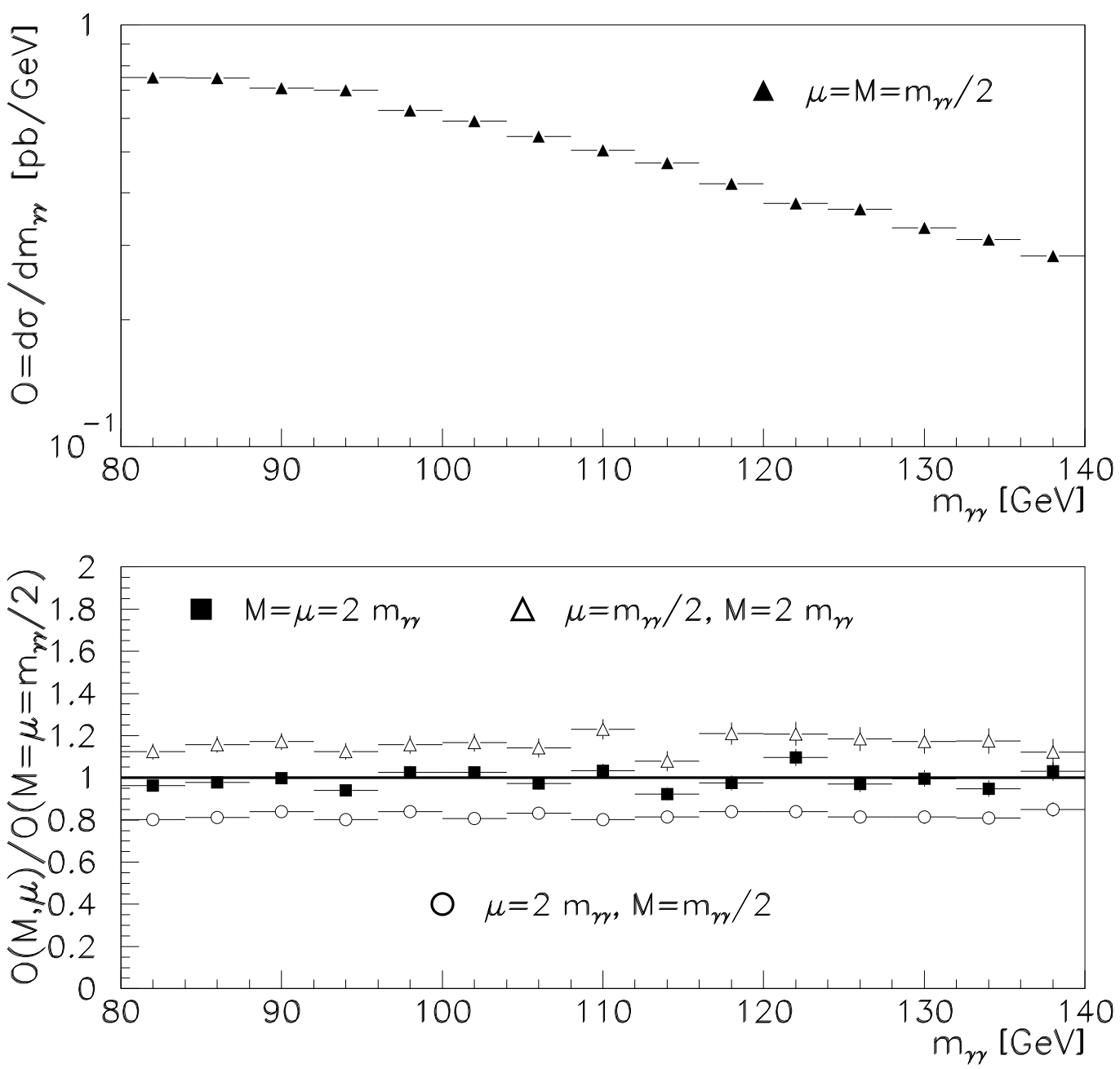

Figure 7: Diphoton differential cross section $d \sigma / d m_{\gamma \gamma}$ vs. $m_{\gamma \gamma}$, the invariant mass of photon pairs, at LHC, $\sqrt{S}=14 \mathrm{TeV}$ without isolation. The following kinematic cuts are applied: $p_{T}\left(\gamma_{1}\right) \geq 40 \mathrm{GeV}$, $p_{T}\left(\gamma_{2}\right) \geq 25 \mathrm{GeV},\left|y\left(\gamma_{1,2}\right)\right| \leq 2.5$. The scale dependence is shown on the bottom plot. $M=M_{f}$ is understood. 

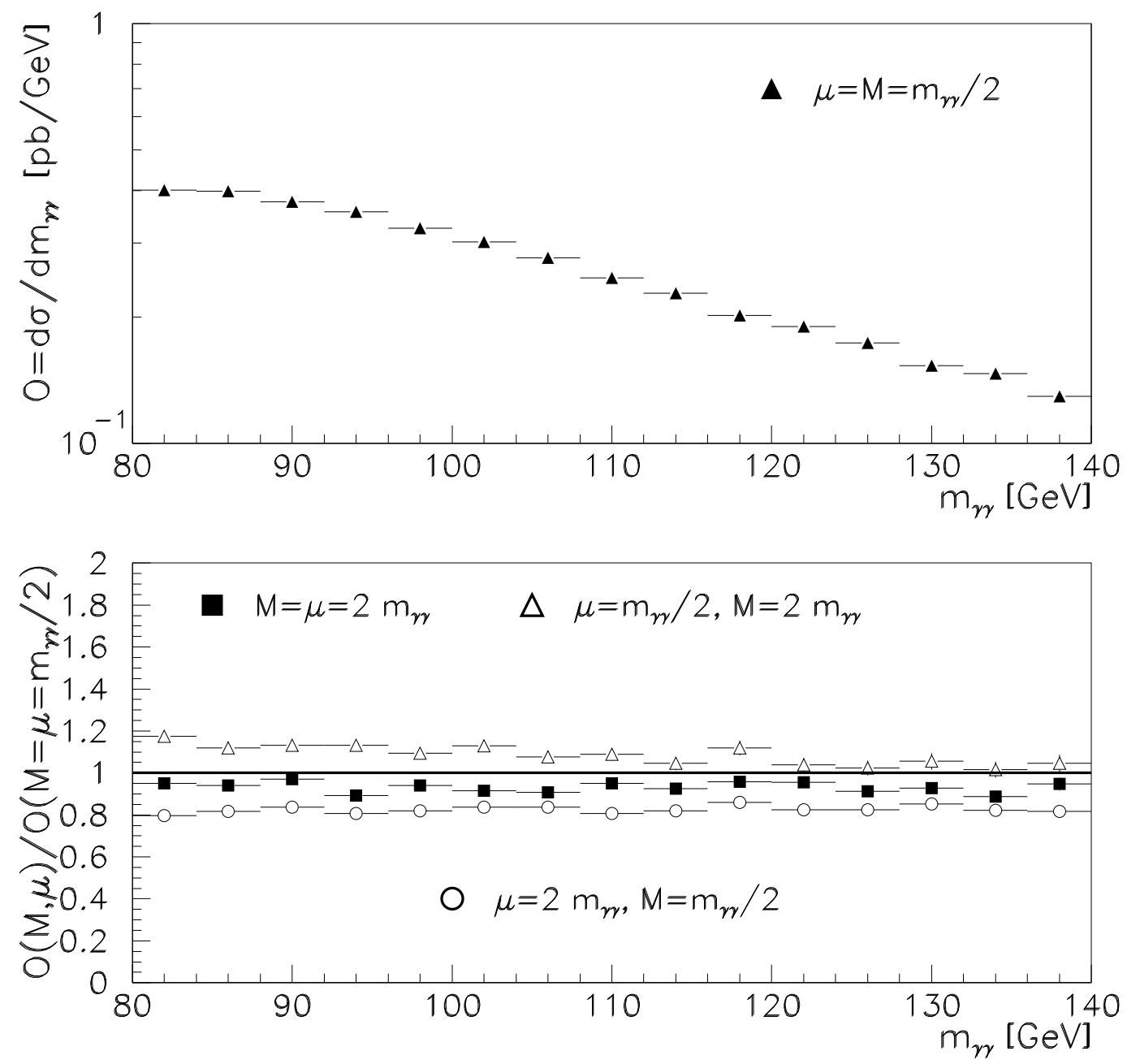

Figure 8: Diphoton differential cross section $d \sigma / d m_{\gamma \gamma}$ vs. $m_{\gamma \gamma}$ at LHC, $\sqrt{S}=14 \mathrm{TeV}$, with isolation criterion $E_{\text {Tmax }}=5 \mathrm{GeV}$ in $R=0.4$. Same kinematic cuts as in fig. 7. The scale dependence is shown on the bottom plot. $M=M_{f}$ is understood. 


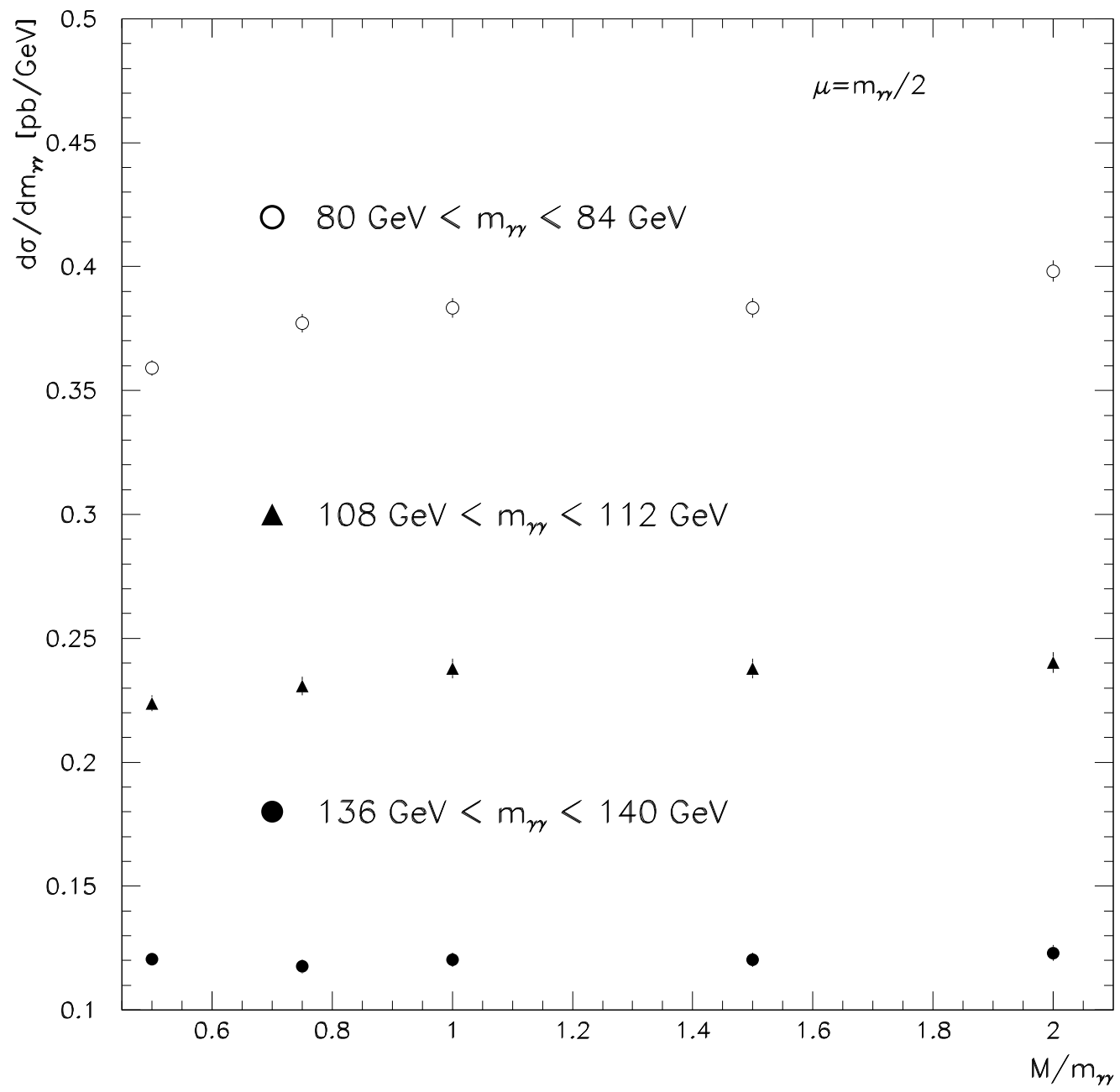

Figure 9: $M$ dependence of the "direct+box" contribution to $d \sigma / d m_{\gamma \gamma}$ in several $m_{\gamma \gamma}$ bins at LHC, $\sqrt{S}=14 \mathrm{TeV}$, with isolation criterion $E_{T \max }=5 \mathrm{GeV}$ in $R=0.4$. Same kinematic cuts as in fig. 7 . $\mu$ is chosen to be $m_{\gamma \gamma} / 2$, while $M$ is varied between $m_{\gamma \gamma} / 2$ and $2 m_{\gamma \gamma}$. 

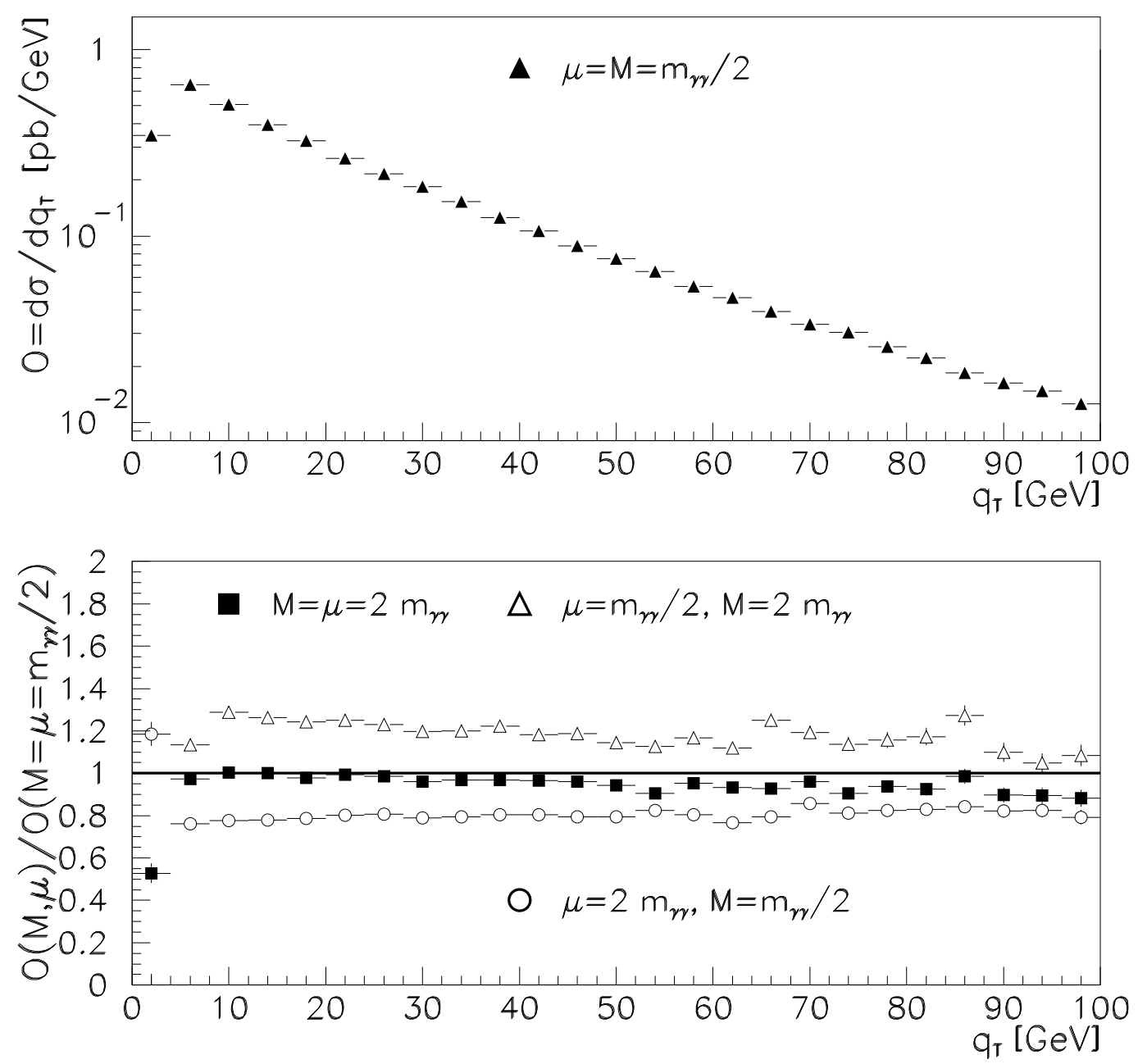

Figure 10: Diphoton differential cross section $d \sigma / d q_{T}$ at LHC, $\sqrt{S}=14 \mathrm{TeV}$, with isolation criterion $E_{\text {Tmax }}=5 \mathrm{GeV}$ in $R=0.4$. The following kinematic cuts are applied: $p_{T}\left(\gamma_{1}\right) \geq 40 \mathrm{GeV}, p_{T}\left(\gamma_{2}\right) \geq 25$ $\mathrm{GeV},\left|y\left(\gamma_{1,2}\right)\right| \leq 2.5$, and $80 \mathrm{GeV} \leq m_{\gamma \gamma} \leq 140 \mathrm{GeV}$. The scale dependence is shown on the bottom plot. $M=M_{f}$ is understood. 

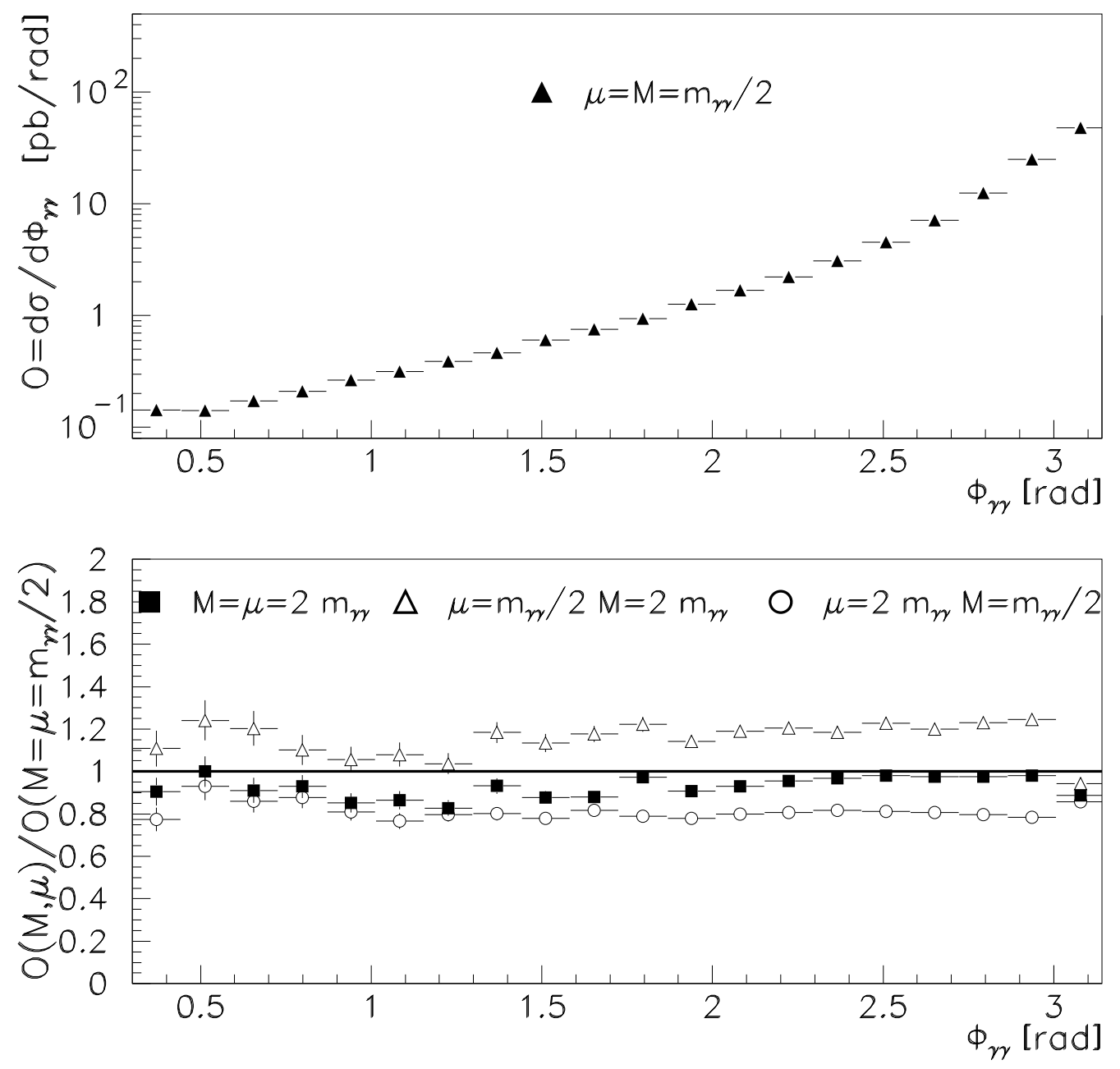

Figure 11: Diphoton differential cross section $d \sigma / d \phi_{\gamma \gamma}$ vs. $\phi_{\gamma \gamma}$, the azimuthal angle between the two photons, at LHC, $\sqrt{S}=14 \mathrm{TeV}$, with isolation criterion $E_{\text {Tmax }}=15 \mathrm{GeV}$ in $R=0.4$. Same kinematic cuts as in fig. 10. The scale dependence is shown on the bottom plot. $M=M_{f}$ is understood. 


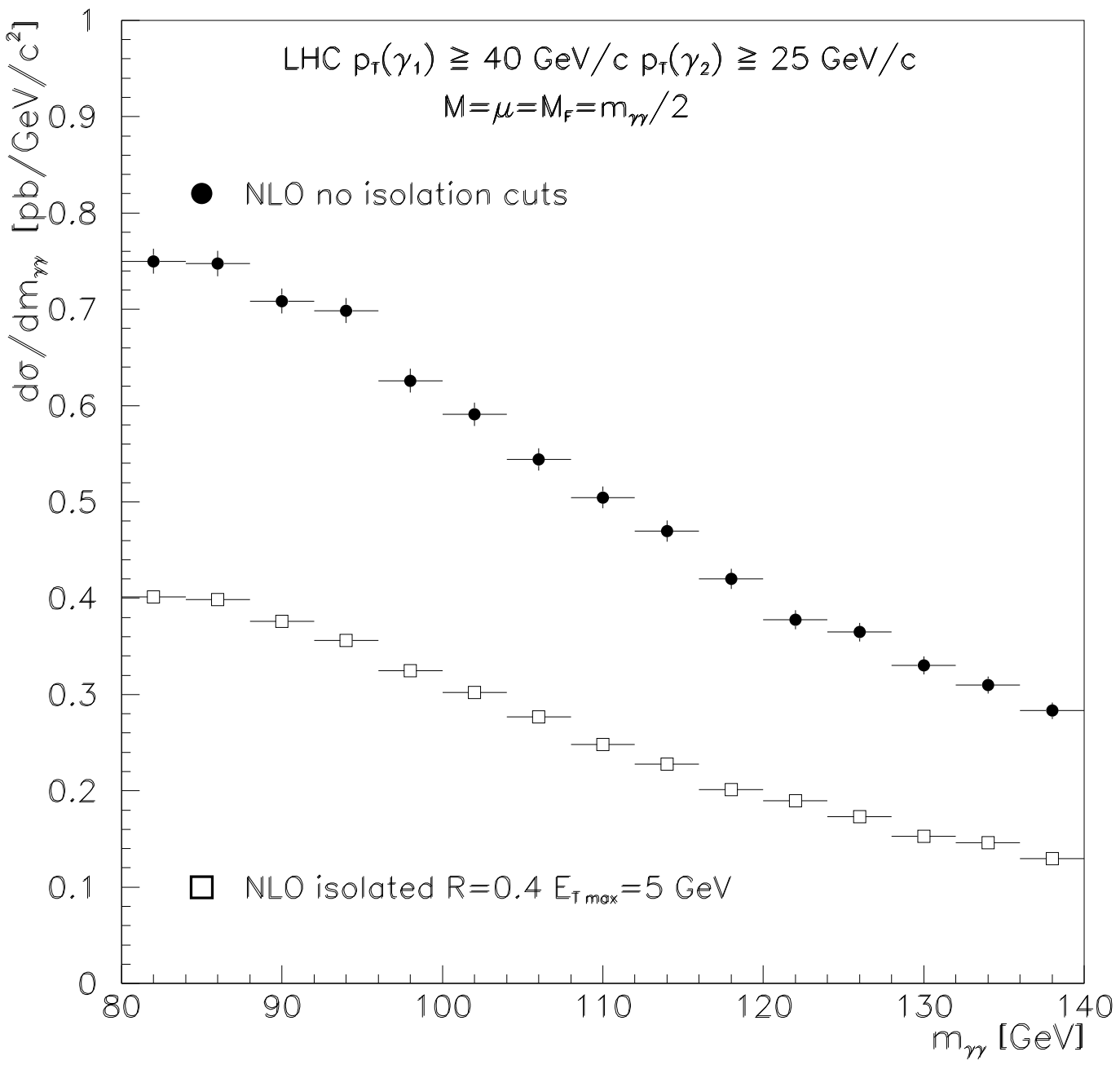

Figure 12: Diphoton differential cross section $d \sigma / d m_{\gamma \gamma}$ vs. $m_{\gamma \gamma}$ at LHC, $\sqrt{S}=14 \mathrm{TeV}$, without and with isolation criterion $E_{\text {Tmax }}=5 \mathrm{GeV}$ in $R=0.4$. Same kinematic cuts as in fig. 7 . The scale choice is $M=M_{f}=\mu=m_{\gamma \gamma} / 2$. 


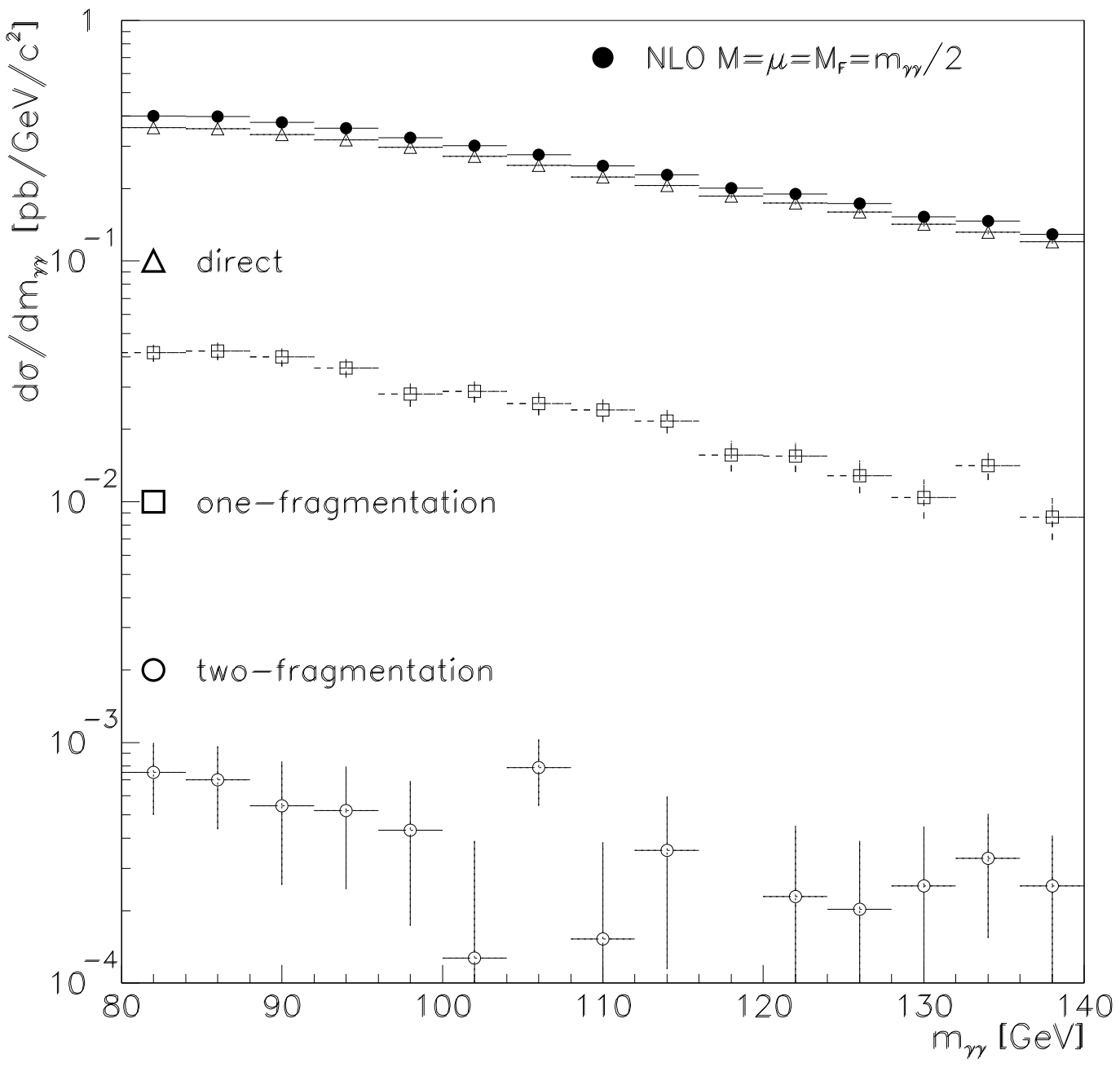

Figure 13: Splitting of the diphoton differential cross section $d \sigma / d m_{\gamma \gamma}$ at LHC, $\sqrt{S}=14 \mathrm{TeV}$ with isolation criterion $E_{T \max }=5 \mathrm{GeV}$ in $R=0.4$, into the "direct", "one fragmentation" and "two fragmentation" components, shown for the scale choice $\mu=M=M_{f}=m_{\gamma \gamma} / 2$. The following kinematic cuts are applied: $p_{T}\left(\gamma_{1}\right) \geq 40 \mathrm{GeV}, p_{T}\left(\gamma_{2}\right) \geq 25 \mathrm{GeV},\left|y\left(\gamma_{1,2}\right)\right| \leq 2.5$. 


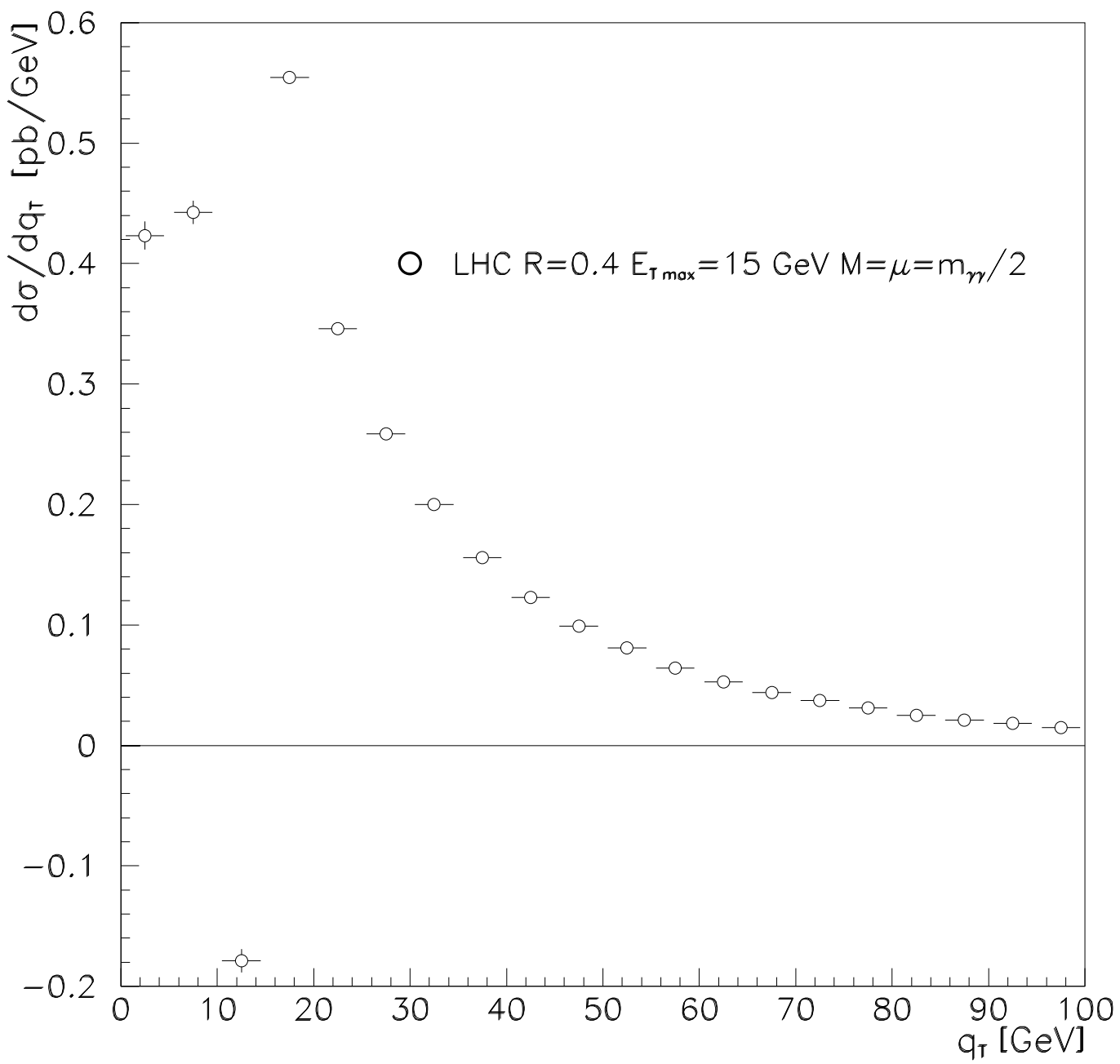

Figure 14: Diphoton differential cross section $d \sigma / d q_{T}$ at LHC, $\sqrt{S}=14 \mathrm{TeV}$, with isolation criterion $E_{\text {Tmax }}=15 \mathrm{GeV}$ in $R=0.4$. Same kinematic cuts as in fig. 0 . 